\title{
Osteologia craniana de Platalea ajaja (Linnaeus) (Aves, Ciconiiformes), comparada com outras espécies de Threskiornithidae
}

\author{
Carolina D. Ferreira ${ }^{1} \&$ Reginaldo J. Donatelli ${ }^{2}$ \\ 1 Programa de Pós-Graduação em Zoologia, Instituto de Biociências, Universidade Estadual Paulista. Rubião Júnior, \\ 18618-100 Botucatu, São Paulo, Brasil. E-mail: fdcarol@yahoo.com.br \\ ${ }^{2}$ Departamento de Ciências Biológicas, Faculdade de Ciências, Universidade Estadual Paulista. Caixa Postal 473, \\ 17001-970 Bauru, São Paulo, Brasil.E-mail: rjdonat@uol.com.br
}

\begin{abstract}
Skull osteology of Platalea ajaja (Linnaeus) (Aves, Ciconiiformes), compared with others species of Threskiornithidae. Platalea ajaja (Linnaeus, 1758) belongs to the Threskiornithidae (ibises and spoonbills), Threskiornitoidea, Ciconiiformes. The Threkiornithidae are currently divided into two groups: Threskiornithinae (ibises and allies), which have curved bills used to probe into the mud, and Plataleinae that have specialized flat bill shapes. The goal of the present work was to describe the cranial osteology of P. ajaja (Plataleinae) and compare it with other species of the family Threskiornithidae. Other species pertaining to the order Ciconiiformes were also considered for analyses and comparison with the former one. The results showed that $P$. ajaja presents many distinct characteristics from others species of the same family as: the shape of the upper jaw, dorsoventrally flattend, larger on the maxillar surface, taking a characteristic shape of a spoon; a conspicuous frontonasal suture; a short supraorbital process of the lacrimal bone; a conspicuous and sharp lateral process of the paraesphenoid bone; the nares are short with an oval shape; a short retroarticular process of the mandible; a short projection of the parasphenoid bone; a dorsoventrally flattened mandible. The characteristics shared between P. ajaja and other Threskiornithidae are: frontal and lacrimal bones not fused; well developed lacrimal bone, zigomatic and paroccipital processes; occipital fonticuli present; nares located in a ridge of the premaxillar bone; reduced maxillar bone; a conspicuous and laterally flattened vomer. However, it is necessary more osteological studies with other species of the same family and a phylogenetic analysis of the group for better understanding of the relationships among the Threskiornithidae. KEY WORDS. Anatomy, birds, Ibis, Roseate Spoonbill, skull.
\end{abstract}

RESUMO. Platalea ajaja (Linnaeus, 1758) pertence Threskiornithidae (curicacas e afins e colhereiros), Threskiornithoidea, Ciconiiformes. Threskiornithidae (colhereiros, curicacas e afins) são subdivididos em dois grupos: Threskiornithinae que compreende curicacas e afins, caracteriza-se por seus representantes possuírem um bico longo, estreito e curvo, ao passo que Plataleinae inclui os colhereiros, que apresentam bico longo e achatado na ponta. O presente trabalho teve como objetivo descrever a osteologia craniana de P. ajaja (Plataleinae) e comparar com outras espécies pertencentes à Threskiornithinae. As espécies utilizadas para tal comparação foram: Mesembrinibis cayennensis (Gmelin, 1789), Phimosus infuscatus (Lichteinstein, 1823), Theristicus caudatus (Boddaert, 1783) e Plegadis chivi (Vieillot, 1817). Outras espécies pertencentes a ordem Ciconiiformes também foram utilizadas para comparação. A partir do presente estudo, observou-se que P. ajaja (Plataleinae) apresenta como características que a separa dos Threskiornithinae: 1) sutura frontonasal conspícua; 2) processo supra-orbital do osso lacrimal curto; 3) maxila superior achatada dorsoventralmente, sendo alargada na extremidade, tomando forma de uma colher; 4) narina curta e de forma oval; e 5) processo retroarticular curto. Entretanto, são necessários estudos osteológicos que contemplem as outras espécies da família, bem como um estudo filogenético do grupo para maior compreensão das relações existentes entre os Threskiornithidae.

PALAVRAS CHAVE. Anatomia, aves, colhereiros, crânio, curicacas.

Platalea ajaja (Linnaeus, 1758) pertence à Threskiornithidae (curicacas e afins, colhereiros), Ciconiiformes (Monroe \& Sibley 1993). Esta ordem engloba também Ardeidae (garças), Cochleariidae (arapapás), Ciconiidae (cegonhas), Scopidae (aves-cabeça-de-martelo) e Balaenicipitidae (aves-bico-de-sapa- to), sendo os Threskiornithidae mais aparentados aos Ciconiidae que aos Ardeidae (Sick 1997).

O histórico taxonômico da ordem e das famílias como um todo evidencia as inúmeras divergências taxômicas do grupo em questão. Huxley (1867) acreditava que os Threskiornithidae

Revista Brasileira de Zoologia 22 (3): 529-551, setembro 2005 
eram mais relacionados aos flamingos. Sundevall (1872) colocou as garças, colhereiros, cegonhas, curicacas, aves-cabeça-de-martelo e aves-de-bico-de-sapato, assim como outros Gruiformes e saracuras em uma ordem denominada Grallatores (correspondente a Grallae de Linnaeus). Reichenow (1882) incluiu na ordem Gressores as famílias Ibidae, Ciconiidae, Phoenicopteridae, Scopidae, Balaenicipitidae e Ardeidae. Os colhereiros, curicacas e afins, de acordo com Sеввонм (1895), pertenciam à Ibididae, subordem Herodiones, ordem Ciconiiformes. GADOW (1893) incluiu os colhereiros, curicacas e afins em Ibidae, ordem Ciconiiformes. Segundo Sharpe (1899), a ordem Ardeiformes era dividida nas subordens Plataleae (colhereiros, curicacas e afins), Ciconiae (cegonhas), Scopi (membros de Scopidae), Balaenicipites (Balaenicipidae) e Ardeae (garças). Já Mrtchell (1913) agrupou membros de Balaenicipitidae com as cegonhas, curicacas e afins e o gênero Scopus dentro da subordem Ciconiae e as garças, na subordem Ardeae. Verheyen (1961) incluiu Scopidae, Ciconiidae e Threskiornithidae na ordem Ciconiiformes. Sibley \& AHLQUist (1972) compararam padrões de eletroforese de proteínas da clara de ovos dos membros dos Ciconiiformes, exceto Balaeniceps (Gould, 1850). De acordo com estes autores, as cegonhas, as curicacas e afins pouco diferem das garças e entre si; os flamingos foram considerados mais próximos dos Ciconiiformes. Segundo KAHL (1979), os colhereiros pertencem à Plataleinae e as curicacas e afins à Threskiornithinae, estando estas compreendidas em Threskiornithidae, ordem Ciconiiformes. Já CRACRAFT (1981) define duas linhagens em Ciconiiformes: 1) flamingos, cegonhas, colhereiros, curicacas e afins; e 2) garças e Balaeniceps. Estes dois grupos são considerados como grupo-irmão de Scopus.

Atualmente, os Threskiornithidae são subdivididos em dois grupos facilmente distinguíveis por suas características externas, sendo mais comumente considerados como duas subfamílias. A subfamília Threskiornithinae compreende as curicacas e afins, que se caraterizam por possuirem um bico longo, estreito e curvo, ao passo que Plataleinae é formada pelos colhereiros, que apresentam bico longo e achatado na ponta, em forma de colher (MATHeu \& DEL Hoyo 1992). Esta subdivisão também é adotada por Austin \& Singer (1983), AMERICAN Ornithologists Union (1998) e Howard \& Moore (1998).

O objetivo deste trabalho foi primariamente descrever a osteologia craniana de P. ajaja (Plataleinae), observar a sua organização e secundariamente, compará-la com algumas espécies de Threskiornithinae. As questões impostas foram as seguintes: a) como está organizada a osteologia craniana de P. ajaja?; e b) quais são as semelhanças e diferenças entre a osteologia craniana de $P$. ajaja e das outras espécies de Threskiornithidae?

\section{MATERIAL E MÉTODOS}

O presente estudo foi realizado com base em 18 crânios de representantes pertencentes à família Threskiornithidae, incluídos em quatro gêneros. Posteriormente foi realizada uma comparação com algumas espécies pertencentes aos Ciconiiformes, baseando-se em crânios de 22 espécimens pertencentes à
Ardeidae, Ciconiidae, Cathartidae e Phoenicopteridae.

O material utilizado Para o estudo pertence ao acervo da coleção anatômica (osteológica) do Museu Paraense Emílio Goeldi, Belém, Pará, Brasil (MPEG).

Segue-se abaixo a relação do material estudado:

Threskiornithidae, Plataleinae: Platalea ajaja MPEG n¹466 (macho) (desenho), MPEG n509 (fêmea), MPEG n 704 (macho), MPEG nº1402 (fêmea), MPEG n¹660 (fêmea), MPEG $\mathrm{n}^{\circ} 1670$ (fêmea).

Threskiornithinae: Mesembrinibis cayennensis (Gmelin, 1789) MPEG n604 (fêmea) (desenho), MPEG n¹272 (macho), MPEG n¹529 (fêmea), MPEG n¹640 (fêmea); Eudocimus ruber (Linnaeus, 1758) MPEG $\mathrm{n}^{\circ} 327$ (fêmea), MPEG $\mathrm{n}^{\circ} 400$ (macho), MPEG n603 (macho), MPEG n¹991 (macho) (desenho); Theristicus caudatus (Boddaert, 1783) MPEG $\mathrm{n}^{\circ} 797$ (macho), MPEG n¹020 (macho), MPEG n¹080 (macho), MPEG n¹399 (fêmea) (desenho).

Ardeidae: Ardea cocoi Linnaeus, 1766 MPEG nº1631 (macho), MPEG n¹689 (?); Tigrisoma lineatum (Boddaert, 1783) MPEG n³66 (macho), MPEG n¹532 (fêmea); Ardea alba Linnaeus, 1758 MPEG $n^{\circ} 463$ (fêmea), MPEG n¹451 (?); Butorides striatus (Linnaeus, 1758) MPEG n671 (fêmea), MPEG n674 (macho); Nycticorax nycticorax (Linnaeus, 1758) MPEG $n^{\circ} 822$ (?), MPEG n¹064 (macho); Egretta thula (Molina, 1782) MPEG n702 (fêmea), MPEG n927 (macho).

Ciconiidae: Ciconia maguari (Gmelin, 1789) MPEG n¹67 (macho), MPEG n¹224 (fêmea); Mycteria americana Linnaeus, 1758 MPEG n²112 (?), MPEG n²174 (macho); Jabiru mycteria (Lichtenstein, 1819) MPEG n988 (macho), MPEG $\mathrm{n}^{\circ} 2182$ (macho).

Cathartidae: Coragyps atratus Bonnaparte, 1780 MPEG nº34 (macho), MPEG n963 (macho).

Phoenicopteridae: Phoenicopterus ruber Linnaeus, 1758 MPEG n²00 (?), MPEG n²04 (?).

Os crânios foram observados utilizando-se microscópio estereoscópio ZEISS SV11 (ocular $10 \mathrm{x}$ e objetivas variando entre $0,8-5,0 \mathrm{x})$.

As descrições osteológicas de $P$. ajaja foram utilizadas como um referencial para as comparações das diversas estruturas anatômicas com as outras espécies de Threskiornithidae, sendo que as observações nestas espécies foram feitas apenas quando se apresentaram distintas das encontradas em P. ajaja.

Por haver fusão entre a maioria dos ossos do crânio e da mandíbula nas aves, nem sempre se pode caracterizar os limites ósseos de algumas estruturas. Assim, muitas estruturas foram aqui descritas utilizando-se limites determinados por regiões, como as propostas por JolLIE (1957) e também utilizada por HöFLING \& GASC (1984).

Todo material osteológico foi desenhado, ilustrado em pranchas em vistas dorsal, ventral, lateral e caudal e nomeados apenas os detalhes mais relevantes ao presente estudo.

Os desenhos são referentes aos representantes de Threkiornithidae, por serem estes o objetivo do presente estudo. As 
outras espécies estudadas não foram desenhadas, servindo apenas de comparação para a análise.

A nomenclatura utilizada para a descrição da osteologia seguiu àquela proposta pela Nomina Anatomica Avium (BAUMEL \& WitMer 1993); na ausência de nomenclatura para determinadas estruturas, elas foram denominadas por letras como também fora utilizado por HöFLING \& GASC (1984). Para a nomenclatura das espécies seguiu-se Matheu \& Del Hoyo (1992) e as normas do Comitê Brasileiro de Registros Ornitológicos (CBRO).

\section{RESULTADOS E DISCUSSÃO}

\section{Ossa cranii}

\section{Os frontale, os parietale, os lacrimale, os ectethmoidale, os mesethmoidale}

A região do osso frontal (os frontale, F - Figs 1 e 2) articula-se rostromedialmente com o processo frontal do osso premaxilar e rostrolateralmente com o processo frontal do osso nasal. Laterorrostralmente, a região frontal contata-se com o processo supra-orbital (proc. supraorbitalis, PrSo - Fig. 3) do osso lacrimal (os lacrimale, L - Figs 2 e 3) por meio da face articular frontonasal (facies articularis frontonasalis) na região da sutura lacrimofrontal (slf - Fig. 2). Os ossos frontal e lacrimal em $P$. ajaja e nos Threskiornithinae estudados não estão fundidos, assim como nos Ardeidae. Já C. maguari, M. americana e C. atratus apresentam estes ossos fundidos.

Rostralmente, o osso frontal articula-se com a maxila superior por meio de uma sutura frontonasal (sfn - Figs 1 e 2), na zona flexora craniofacial (zona flexoria craniofacialis). A região frontal contata-se ventralmente com o septo interorbital (SI - Fig. 3) e caudalmente, continua-se com o osso parietal (os parietale, $\mathrm{P}$ - Figs 1 e 2), formando conjuntamente com este, o teto craniano.

Nos Threskiornithinae, a região do osso frontal fusionada ao nasal apresenta uma concavidade localizada rostromedialmente, que é mais profunda em E. ruber (C1 - Fig. 4), seguida por T. caudatus (C1 - Fig. 6) e M. cayennensis (C1 Fig. 5) onde é mais rasa; está ausente em $P$. ajaja. Tal concavidade presente nos Threskiornithinae parece ser uma característica que os separam dos Plataleinae. Esta concavidade também é observada em C. maguari e nos Ardeidae estudados, onde se extende da metade do osso frontal em direção a região da zona flexora craniofacial. Está ausente em M. americana, Jabiru mycteria, C. atratus e P. ruber.

Não se observa a sutura frontonasal nos Threskiornithinae estudados, sendo apenas observada em $P$. ajaja. Também não é observada em A. alba, B. striatus, E. thula, N. nycticorax, C. maguari e $P$. ruber.

A região parietal ( $\mathrm{P}$ - Figs 1-3) limita-se caudolateralmente com a fossa temporal caudal (fossa temporalis caudalis, FTc Fig. 3) da região esquamosal, por meio da crista temporal (crista temporalis, CrT - Fig. 3) e caudalmente com a região occipital, por meio da conspícua crista nucal transversa (crista nuchalis transversa, CrNT - Fig. 3).
O osso lacrimal (os lacrimale, L - Fig. 3) é composto por um corpo achatado rostrocaudalmente formando uma lâmina fina, onde se distingue as seguintes partes: a) Processo supra-orbital (Proc. supraorbitalis, PrSo - Fig. 3), desenvolvido nesta espécie, limita-se com os ossos nasal e frontal por meio da face articular frontonasal; b) Processo 1 (Pr1 - Fig. 3), curto e localizado lateralmente no corpo do osso lacrimal; c) Processo 2 (Pr2 - Fig. 3), conspícuo, curto e afilado em sua extremidade, situado rostromedialmente no corpo do lacrimal; e d) Processo orbital (proc. orbitalis, PrO - Fig. 3), denominado pé do lacrimal por outros autores (ver CRACRAFT 1968); projeta-se rostroventralmente, não atingindo o arco jugal. Há uma variação individual nesta espécie, pois em alguns exemplares, inclusive em um mesmo espécime, o processo orbital mostra-se ora bífido, ora sem qualquer bifurcação.

Em P. ajaja, o processo supra-orbital (PrSo - Fig. 3) do lacrimal é curto, sendo que nos Threskiornithinae (PrSo - Figs 7, 8 e 9) estudados, ele se apresenta mais longo; trata-se de uma característica que separa os Plataleinae dos Threskiornithinae. Este processo é mais longo e muito bem desenvolvido em Threristicus caudatus (PrSo - Fig. 9), seguido por M. cayennensis (PrSo - Fig. 8) e E. ruber (PrSo - Fig. 7). A. cocoi, T. lineatum, A. alba, B. striatus e $E$. thula possuem este processo mais desenvolvido que $P$. ajaja, seguidos por J. mycteria e N. nycticorax. Em C. maguari, M. americana e $C$. atratus, ele se funde ao osso frontal.

De acordo com Cracraft (1968), o lacrimal articula-se somente com o nasal nos Threskiornithidae, exceto em Ajaia (Linnaeus, 1758) ( = Platalea Linnaeus, 1758) e Nipponia (Temminck, 1835); todavia, observa-se claramente o lacrimal contatando-se com o frontal e o nasal tanto em $P$. ajaja como em M. cayennensis. Já em E. ruber e T. caudatus o lacrimal contatase somente com o nasal, corroborando com a afirmação de Cracraft (1968), quanto a estas duas espécies.

O processo supra-orbital do lacrimal contata-se tanto com o frontal quanto com o nasal em P. ajaja (L, N e F - Fig. 2) e em M. cayennensis (L, N e F - Fig. 5); já em Threristicus caudatus (L e $\mathrm{N}$ - Fig. 6) e E. ruber (L e N - Fig. 4), este processo contata-se somente com o osso nasal.

O processo 1 do osso lacrimal é curto em P. ajaja (Pr1 Fig. 3), atingindo seu desenvolvimento máximo em Threristicus caudatus (Pr1 - Fig. 9), sendo menos desenvolvido em $M$. cayennensis ( $\operatorname{Pr} 1$ - Fig. 8), seguido por E. ruber (Pr1 - Fig. 7) que se assemelha muito ao de $P$. ajaja. Os Ciconiidae estudados, bem como $C$. atratus e $P$. ruber e a maioria dos Ardeidae não apresentam este processo, com exceção de T. lineatum, onde o processo 1 é desenvolvido e dirige-se ventralmente e não lateralmente como em P. ajaja (Pr1 - Fig. 3).

Já o processo 2 é curto e afilado em P. ajaja (Pr2 - Fig. 3), porém é mais longo em M. cayennensis (Pr2 - Fig. 8) e Threristicus caudatus (Pr2 - Fig. 9) onde se apresenta largo da base até a sua extremidade; em E. ruber (Pr2 - Fig. 7) é extremamente curto. Este processo está ausente nas espécies estudadas de Ardeidae, Ciconiidae, em P. ruber e C. atratus. 


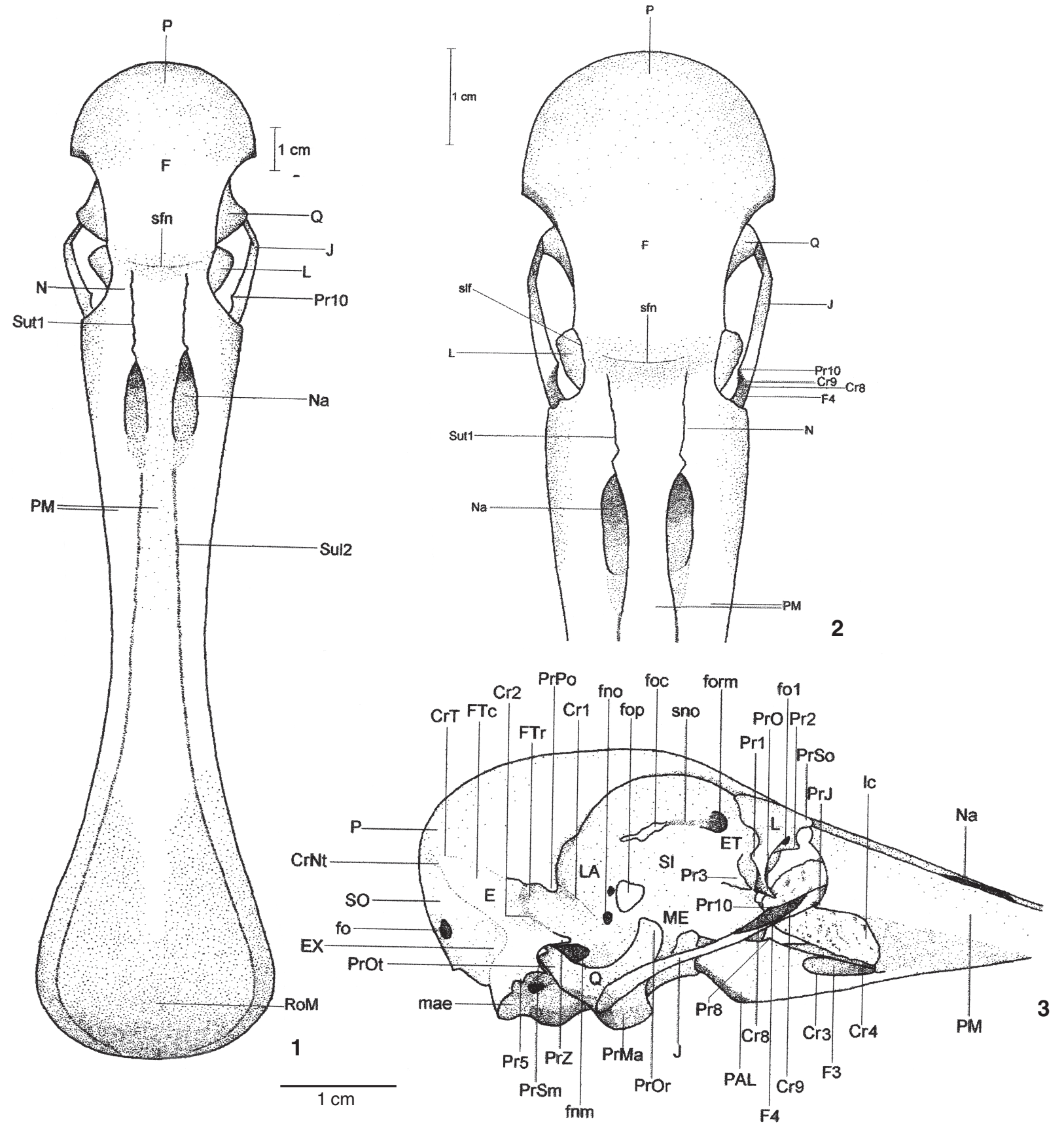

Figuras 1-3. Crânio de Platalea ajaja: (1-2) vista dorsal; (3) vista lateral. (Cr1-Cr9) Crista 1 a crista 9, (CrNt) crista nucal transversa, (CrT) crista temporal, (E) esquamosal, (ET) ectetmóide, (EX) exoccipital, (F) frontal, (F3) fossa 3, (F4) fossa 4, (fnm) forâmen do nervo maxilomandibular, (fno) forâmen do nervo oftálmico, (fo) fontículo occipital, (fo1) forâmen 1, (foc) fontículo orbitocranial, (fop) forâmen óptico, (form) forâmen orbitonasal medial, (FTc) fossa temporal caudal, (FTr) fossa temporal rostral, (J) arco jugal, (L) lacrimal, (LA) lateroesfenóide, (Ic) lamela coanal, (mae) meato acústico externo, (ME) mesetmóide, (N) nasal, (Na) narina, (P) parietal, (PAL) palatino, (PM) premaxilar, (Pr1) processo 1, (Pr10) processo 10, (Pr2-Pr8) processo 2 a (Pr8) processo 8, (Prj) processo jugal do maxilar, (PrMa) processo mandibular do quadrado, (PrO) processo orbital do lacrimal, (PrOr) processo orbital do quadrado, (PrOt) processo ótico o quadrado, (PrPo) processo pós-orbital, (PrSm) processo suprameático, (PrSo) proceso supra-orbital, (PrZ) processo zigomático, (Q) quadrado, (RoM) rostro maxilar, (sfn) sutura frontonasal, (SI) septo interorbital, (slf) sutura lacrimofrontal, (sno) sulco do nervo olfatório, (SO) supraoccipital, (Sul 2) sulco 2, (Sut 1 ) sutura 1. 
Ainda em relação ao lacrimal, o processo orbital é pouco desenvolvido em Threristicus caudatus (PrO - Fig. 9) com menos metade do comprimento desde a sua origem até o arco jugal. Já em E. ruber (PrO - Fig. 7) e M. cayennensis (PrO - Fig. 8) este processo mostra-se mais longo e afilado na sua extremidade que em P. ajaja (PrO - Fig. 3). Todavia, não atinge o arco jugal, assim como em P. ajaja (PrO - Fig. 3). Este processo é bem desenvolvido nos Ardeidae estudados e orienta-se caudalmente, sendo bífido em $T$. lineatum e $A$. alba, onde a porção lateral é maior que a medial; em $B$. striatus e $E$. thula há uma variação individual, podendo apresentar-se bífido em alguns indivíduos, onde em $E$. thula a porção medial é maior que a lateral.Em N. nycticorax este processo é bem desenvolvido e de forma retangular na extremidade.

Em C. maguari e J. mycteria o processo orbital apresentase achatado lateromedialmente, sendo que em $C$. maguari orienta-se ventralmente, é longo e afilado na extremidade, em $J$. mycteria orienta-se ventrocaudalmente; este processo é arredondado em M. americana e orienta-se ventralmente. Em C. atratus, o processo orbital dirige-se lateralmente; $P$. ruber também apresenta este processo achatado lateralmente

A incisura do duto nasolacrimal (incisura ductus nasolacrimale) localiza-se rostralmente no corpo do osso lacrimal de P. ajaja; tem forma de fenda característica e possui na sua face rostral um forâmen (fo1 - Fig. 3) localizado dorsomedialmente. Esta incisura é pouco conspícua em E. ruber, M. cayennensis e Threristicus caudatus. Contudo, observa-se somente nos Ciconiidae estudados e não nos demais grupos. O forâmen localizado na face rostral do corpo do lacrimal está presente nos Ciconiidae estudados e em C. atratus, assim como em P. ajaja (fo1 - Fig. 3).

Segundo Cracraft (1968), o lacrimal dos Threskiornithidae possui, além do processo supra-orbital, outros três processos: o primeiro que se orienta medialmente em direção ao ectetmóide (refere-se ao processo 2, no presente estudo); o segundo, menor que o primeiro e se dirige lateralmente (processo 1); e o terceiro que se orienta ventralmente (processo orbital). Esta afirmação corrobora com o que foi observado nos Threskiornithidae, sendo estes processos bastante distintos nas espécies estudadas.

Payne \& Risley (1976) relatam que o lacrimal dos Ardeidae pode ser pequeno, com menos da metade da extensão de sua origem na caixa craniana até o arco jugal; com tamanho médio, com pouco mais da metade desta extensão; e grande, onde alcança o arco jugal e com ele se articula. De acordo com Verheyen (1959), o corpo do lacrimal atinge seu máximo desenvolvimento entre os Ardeidae, mas também é desenvolvido entre Phoenicopteridae, Ciconiidae e Threskiornithidae. No presente estudo observou-se que o lacrimal dos Threskiornithidae é muito bem desenvolvido, principalmente quanto ao processo supra-orbital que é mais desenvolvido nos Threskiornithinae que em $P$. ajaja (Plataleinae), tratando-se de uma característica que separa estas duas subfamílias.

O osso ectetmóide (os ectethmoidale, ET - Fig. 3) compõe a porção rostrolateral da órbita. Apresenta um longo e conspícuo processo (Pr3 - Fig. 3), que se projeta lateralmente aproximando-se da porção medial do processo orbital (proc. orbitalis lacrimalis, PrO - Fig. 3).

O processo 3 do osso ectetmóide encontra o processo orbital em M. cayennensis (Pr3 e PrO - Fig. 8) e Threristicus caudatus (Pr3 e PrO - Fig. 9), situando-se ventralmente a este. Este processo apresenta variação em E. ruber $(\mathrm{Pr} 3$ e $\mathrm{PrO}$ - Fig. 7), podendo ser reduzido ou ainda longo, alcancando o processo orbital; já em P. ajaja (Pr3 e PrO - Fig. 3), o processo 3 apenas se aproxima do processo orbital.

Nos Ardeidae estudados, o processo 3 é desenvolvido, com exceção de T. lineatum onde se apresenta mais curto que nos outros representantes; em $A$. alba e $B$. striatus este processo é achatado rostrocaudalmente e não se afila na extremidade, podendo variar a forma em A. alba; em B. striatus, este processo orienta-se ventralmente. E. thula e N. nycticorax apresentamno também achatado rostrocaudalmente, direcionando-se ventralmente e afilado na sua extremidade, sendo que em $N$. nycticorax este processo é mais desenvolvido que em $E$. thula. Entre os Ciconiidae estudados, C. maguari e J. mycteria apresentam este processo achatado laterolateralmente e orientando-se ventralmente, sendo estreito em J. mycteria; apenas um indivíduo de C. maguari observou-se este processo. M. americana apresenta-o reduzido; já em $C$. atratus este processo se funde com a porção medial do lacrimal. Está ausente em $P$. ruber.

Existe ainda uma projeção rostrodorsal do osso ectetmóide em E. ruber (Pj1 - Fig. 7), Threristicus caudatus (Pj1 Fig. 9) e M. cayennensis (Pj1 - Fig. 8), mas ela está ausente em $P$. ajaja. Esta projeção localiza-se dorsalmente ao processo 3 ( $\mathrm{Pj} 1$ e Pr3 - Figs 7-9) e dirige-se rostralmente, sendo largo da base até a extremidade. Trata-se de uma característica observada somente em Threskiornithinae.

Somente em Threristicus caudatus observa-se o forâmen caudal do corpo do ectetmóide (foEt - Fig. 9), localizado caudomedialmente.

O processo 3 também foi relatado por Cracraft (1968), já a projeção 1 do ectetmóide não foi constatada por nenhum autor; esta projeção 1 está presente somente nos Threskiornithinae, sendo esta outra característica que os separa dos Plataleinae. De acordo com Cracraft (1968), o ectetmóide é bem desenvolvido nos Threskiornithidae, pouco desenvolvido na maior parte dos Ardeidae, ausente em Balaenicipitidae e Scopidae e reduzido ou ausente em Ciconiidae, o que corrobora com o observado por Verheyen (1959). Tal autor (VERHEYen 1959) relata que o osso ectetmóide é bem desenvolvido nos Threskiornithidae, mas se apresenta vestigial nos Ardeidae, Scopidae e certos Ciconiidae (Jabiru Hellmayr, 1906 , Leptoptilos Lesson, 1831) e ausente nos Phoenicopteridae, Balaenicipitidae, alguns Ardeidae e na maior parte dos Ciconiidae. Contudo, observou-se o processo 3 nos Ardeidae e Ciconiidae estudados, com algumas variações; tal fato não foi relatado pelos autores acima citados. 

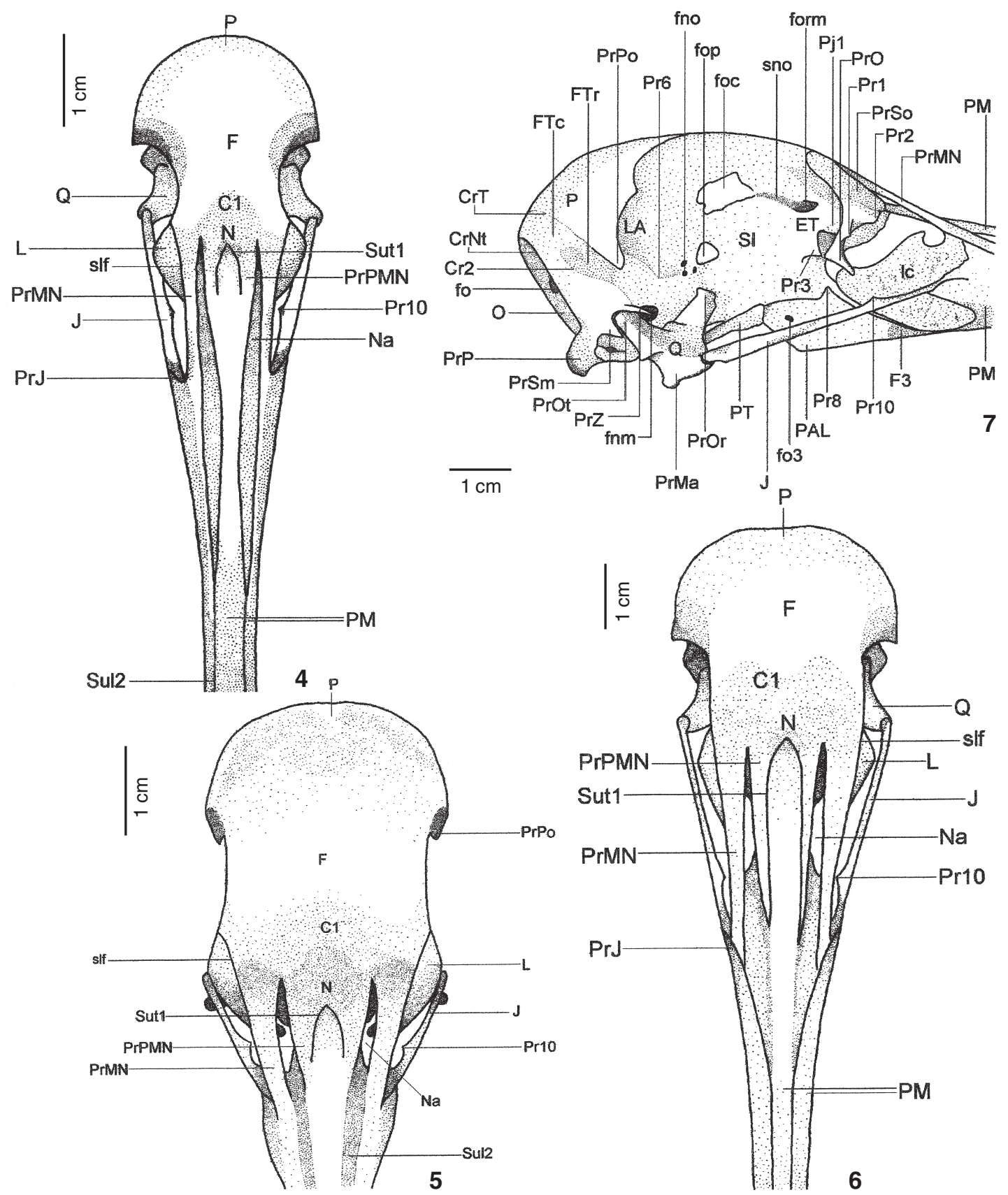

Figuras 4-7. Crânio. (4-6) Vista dorsal: (4) Eudocimus ruber; (5) Mesembrinibis cayennensis; (6) Theristicus caudatus; (7) vista lateral: Eudocimus ruber. (C1) Concavidade 1, (Cr2) crista 2, (CrNt) crista nucal transversa, (CrT) crista temporal, (ET) ectetmóide, (F) frontal, (F3) fossa 3, (fnm) forâmen do nervo maxilomandibular, (fno) forâmen do nervo oftálmico, (fo) fontículo occipital, (fo3) forâmen 3, (foc) fontículo orbitocranial, (fop) forâmen óptico, (form) forâmen orbitonasal medial, (FTc) fossa temporal caudal, (J) arco jugal, (L) lacrimal, (LA) lateroesfenóide, (Ic) lamela coanal, (N) nasal, (Na) narina, (O) occipital, (P) parietal, (PAL) palatino, (Pj1) projeção 1, (PM) premaxilar, (Pr1) processo, (Pr2) processo 2, (Pr3) processo 3, (Pr6) processo 6, (Pr8) processo 8, (Pr10) processo 10, (Prj) processo jugal, (PrMa) processo mandibular do quadrado, (PrMN) processo maxilar do nasal, (PrO) processo orbital do lacrimal, (PrOr) processo orbital do quadrado, (PrOt) processo ótico o quadrado, (PrP) processo paraoccipital, (PrPMN) processo premaxilar do nasal, (PrPo) processo pós-orbital, (PrSm) processo suprameático, (PrSo) proceso supra-orbital, (PrZ) processo zigomático, (PT) pterigóide, (Q) quadrado, (SI) septo interorbital, (slf) sutura lacrimofrontal, (sno) sulco do nervo olfatório, (Sul 2) sulco 2, (Sut 1 ) sutura 1. 


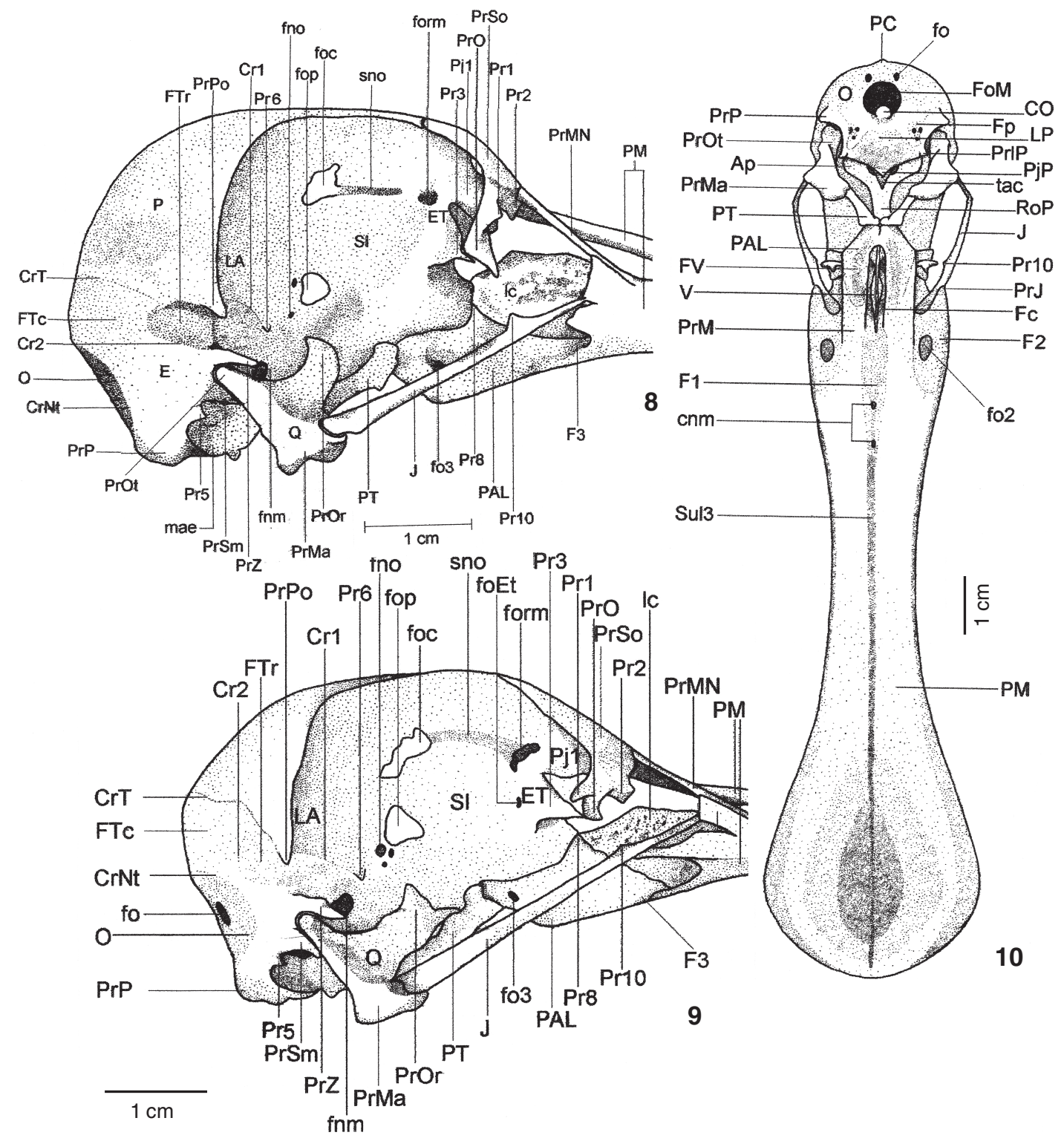

Figuras 8-10. Crânio. (8-9) Vista lateral: (8) Mesembrinibis cayennensis; (9) Theristicus caudatus; (10) vista ventral: Platalea ajaja. (Ap) Ala paraesfenóide, (cnm) canal neurovascular da maxila, (CO) côndilo occipital, (Cr1) crista 1, (Cr2) crista 2, (CrNt) crista nucal transversa, (CrT) crista temporal, (E) esquamosal, (ET) ectetmóide, (F1) fossa 1, (F2) fossa 2, (F3) fossa 3, (Fc) fossa coanal, (fnm) forâmen do nervo maxilomandibular, (fno) forâmen do nervo oftálmico, (fo) fontículo occipital, (fo2) forâmen 2, (fo3) forâmen 3, (foc) fontículo orbitocranial, (foEt) forâmen do ectetmóide, (FoM) forâmen magno, (fop) forâmen óptico, (form) forâmen orbitonasal medial, (Fp) fossa parabasal, (FTc) fossa temporal caudal, (FTr) fossa temporal rostral, (FV) fossa ventral, (J) arco jugal, (LA) lateroesfenóide, (Ic) lamela coanal, (LP) lâmina paraesfenóide, (mae) meato acústico externo, $(\mathrm{O})$ occipital, (P) parietal, (PAL) palatino, (PC) proeminência cerebelar, (Pj1) projeção 1, (PjP) projeção do paraesfenóide, (PM) premaxilar, $(\operatorname{Pr} 1)$ processo 1, (Pr10) processo 10, $(\operatorname{Pr} 2$ processo 2, $(\operatorname{Pr} 3)$ processo 3 , (Pr3) processo 3, (Pr5) processo 5, (Pr6) processo 6, (Pr8) processo 8, (Prj) prcesso jugal, (PrIP) processo lateral do paraesfenóide, (PrM) processo maxilar do palatino, (PrMa) processo mandibular do quadrado, (PrMa) processo mandibular, (PrMN) processo maxilar do nasal, (PrO) processo orbital do lacrimal, (PrOr) processo orbital do quadrado, (PrOt) processo ótico o quadrado, (PrOt) processo ótico, (PrP) processo paraesfenóide, (PrP) processo paraoccipital, (PrPo) processo pós-orbital, (PrSm) processo suprameático, (PrSo) proceso supra-orbital, (PrZ) processo zigomático, (PT) pterigóide, (Q) quadrado, (RoP) rostro do paraesfenóide, (SI) septo interorbital, (sno) sulco do nervo olfatório, (Sul 3) sulco 3, (tac) tuba auditiva comum, (V) vômer. 
O osso mesetmóide (os mesethmoidale, ME - Fig. 3) compõe a maior parte do septo interorbital (SI - Fig. 3) (BAUMEL \& WitMer 1993). Limita-se dorsalmente com o osso frontal onde se situa o sulco do nervo olfatório (sulcus nervus olfactorii, snoFig. 3) que termina no forâmem do nervo olfatório (foramen nervus olfactorii); rostralmente limita-se com o ectetmóide (ET - Fig. 3) e caudalmente com o lateroesfenóide (os laterosphenoidale, LA - Fig. 3), onde se observa o bem desenvolvido forâmen óptico (foramen opticum, fop - Fig. 3).

Nos Threskiornithidae, o forâmen óptico é desenvolvido tanto em P. ajaja (fop - Fig. 3) quanto nos Threskiornithinae (fop - Figs 7-9); entretanto, nos Ardeidae estudados este forâmen é extremamente desenvolvido, ocupando mais da metade do septo interorbital. M. americana e J. mycteria não apresentam tal estrutura.

Nota-se ainda em P. ajaja, entre a região do mesetmóide e o ectetmóide, o conspícuo forâmen orbitonasal medial (form - Fig. 3), de forma arredondada e localizado dorsorrostralmente.

O forâmen orbitonasal medial é mais estreito em Threristicus caudatus (form - Fig. 9) que nos demais Threskiornithidae estudados (form - Figs 3, 7 e 8), onde este se mostra conspícuo e de forma arredondada. Este forâmen é estreito também em $N$. nycticorax, ao contrário dos Ciconiidae estudados e C. atratus onde se assemelha a P. ajaja (form - Fig. 3); está ausente nos demais Ardeidae e $P$. ruber.

O fontículo orbitocranial é mais longo e largo em E. ruber (foc - Fig. 7) que em Threristicus caudatus (foc - Fig. 9) e $M$. cayennensis (foc - Fig. 8). Em P. ajaja (foc - Fig. 3), apresenta-se mais estreito que nas especiés acima citadas. Tal fontículo é bem desenvolvido em A. cocoi, A. alba, B. striatus, E. thula, N. nycticorax e $P$. ruber, estando ausente em T. lineatum, C. atratus e nos Ciconiidae analisados.

\section{Os parasphenoidale, os basisphenoidale, os laterosphenoidale, os squamosum}

Em P. ajaja, o osso paraesfenóide constitui-se de duas porções: a) Rostro paraesfenóide (Rostrum parasphenoidale, RoP - Figs 10 e 11)que corresponde a porção rostral do paraesfenóide, é longa e funde-se dorsalmente ao septo interorbital; compreendendo quase a metade da extensão do osso paraesfenóide. Articula-se rostroventralmente com o osso pterigóide (os pterygoideum, PT - Fig. 11) por meio da face articular pterigóide (facies articularis pterygoidea) e com o osso palatino (os palatinum, PAL Fig. 11) por meio da face articular palatina (facies articularis palatina); e b) Lâmina paraesfenóide (Lamina parasphenoidale, LP - Figs 10 e 11)que se projeta rostralmente, recobrindo parte da porção caudal do rostro paraesfenóide por meio da curta projeção paraesfenóide (PjP - Fig. 11), pouco desenvolvida e que se afila rostralmente. Contata-se caudalmente com o osso basioccipital (os basioccipitale, BA - Fig. 11) por meio da crista basilar transversa (crista basilaris transversa, CrBt - Fig. 11), localizada entre os dois tubérculos basilares (Tb-Fig. 12); caudolateralmente funde-se ao osso exoccipital (os exoccipitale, EX - Fig. 11).

Nos Threskiornithidae, a projeção do paraesfenóide é mais desenvolvida em E. ruber ( $\mathrm{PjP}$ - Fig. 13), M. cayennensis ( $\mathrm{PjP}$ Fig. 14) e Threristicus caudatus ( $\mathrm{Pj} \mathrm{P}$ - Fig. 15) que em $P$. ajaja (PjP - Fig. 11), formando um ápice pontiagudo na sua extremidade. Esta é outra característica associada aos Threskiornithinae. Todavia, os Ardeidae apresentam a projeção paraesfenóide pouco desenvolvida, assim como J. mycteria e C. atratus.

Na face ventral do paraesfenóide, observa-se uma depressão, tuba auditiva comum (tuba auditiva comunis, tac - Fig. 11), localizada rostralmente à projeção paraesfenóide, que forma um triângulo característico junto à ala paraesfenóide (ala parasphenoidale, Ap - Fig. 11).

Esta tuba auditiva comum é longa e bem desenvolvida em M. cayennensis (tac - Fig. 14) e E. ruber (tac - Fig. 13), sendo mais longa que em Threristicus caudatus (tac - Fig. 15) e P. ajaja (tac - Fig. 11). A tuba auditiva comum é bem desenvolvida em A. alba, C. maguari, M. americana e J. mycteria, sendo que em $B$. striatus ela se extende até a metade do rostro paraesfenóide e em C. atratus é mais larga. Tal estrutura está ausente em P. ruber.

A ala paraesfenóide é bem desenvolvida e conspícua em P. ajaja (Ap - Fig. 11) e contribui para a formação da borda rostroventral do meato acústico externo (meatus acusticus externus, mae - Fig. 3). Apresenta dois foramens: canal carótico cranial (canalis caroticus cranialis) e canal orbital (canalis orbitalis). Rostralmente a estes, há um pequeno processo ( $\operatorname{Pr} 4$ Fig. 11), afilado na sua extremidade.

Nos Ardeidae estudados, não foi observada a ala paraesfenóide, que pode estar ausente ou muito reduzida; as demais espécies não apresentam variação significativa nesta estrutura.

O processo 4 do paraesfenóide apresenta-se mais conspícuo em Threristicus caudatus (Pr4 - Fig. 15) e M. cayennensis (Pr4 - Fig. 14) que em P. ajaja (Pr4 - Fig. 11), estando ausente em E. ruber; nas demais espécies estudadas, está presente somente em $C$. atratus, onde é maior, em forma de T e projeta-se laterorrostralmente.

O conspícuo processo lateral do paraesfenóide (proc. lateralis parasphenoidalis, PrlP) projeta-se rostrolateralmente em P. ajaja (PrlP - Fig. 11), afilando-se na extremidade e participa da formação da borda ventral do meato acústico externo (mae - Fig. 3).

O processo lateral paraesfenóide é reduzido ou ainda ausente em E. ruber, M. cayennensis e Threristicus caudatus, mas conspícuo e desenvolvido em P. ajaja (PrlP - Fig. 11); é curto nos Ciconiidae e ausente nos Ardeidae e C. atratus.

Em P. ajaja, a região do osso esquamosal (E - Fig. 3) limita-se rostralmente com o osso lateroesfenóide (LA - Fig. 3) por meio da crista 1 (Cr1 - Fig. 3); dorsocaudalmente limita-se com o osso parietal (P - Fig. 3) por meio da crista temporal (CrT Fig. 3) e caudalmente com a região occipital por meio da crista nucal transversa (CrNt - Fig. 3). Está delimitada ventralmente pela borda dorsal do meato acústico externo (mae - Fig. 3).

Esta região apresenta: a) Processo zigomático (proc. zygomaticus, PrZ - Fig. 3) curto e afilado, com cerca de $1 / 4$ da distância de sua origem até o arco jugal, projetando-se 

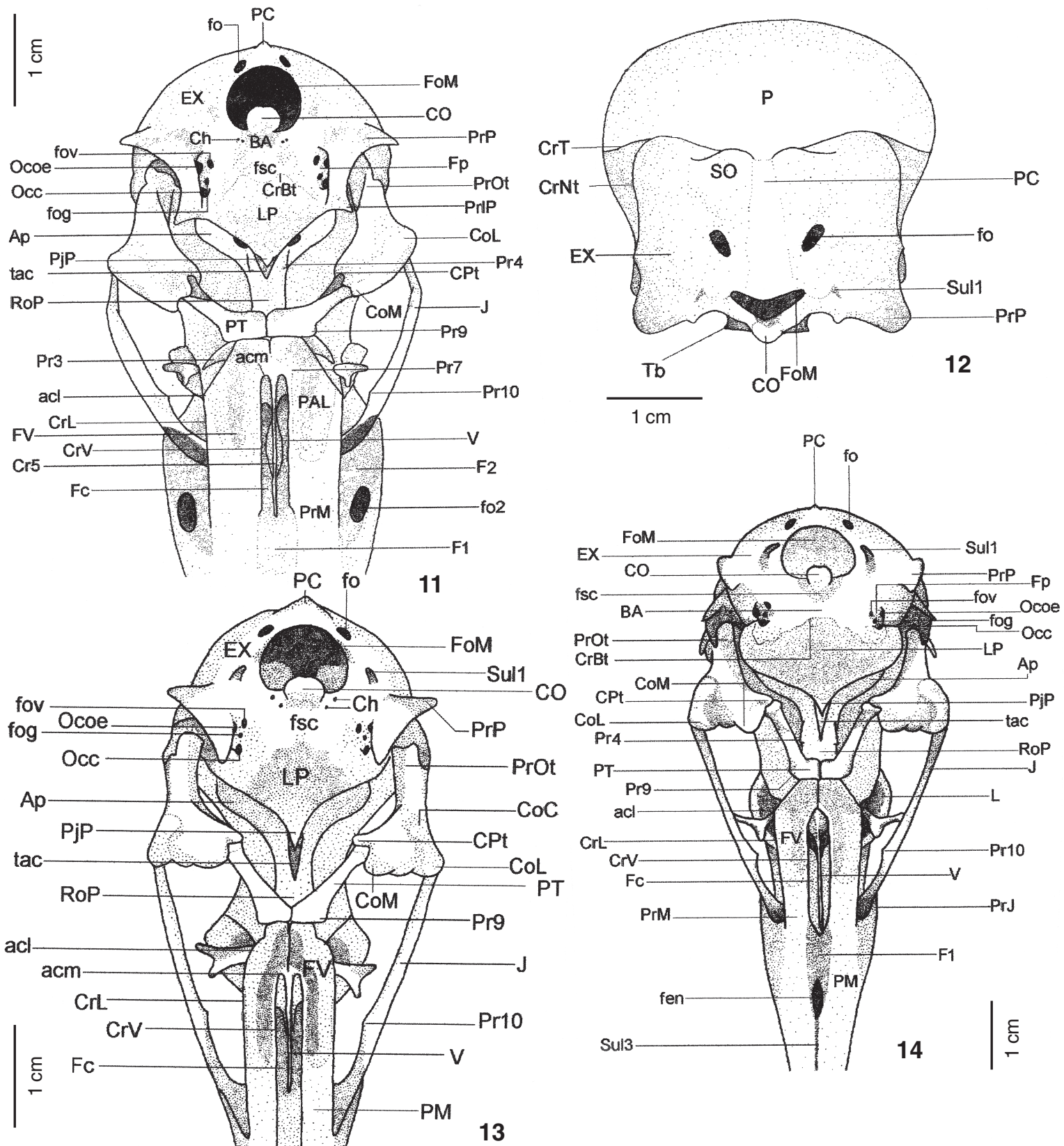

Figuras 11-14. Crânio. (11) Platalea ajaja, vista ventral; (12) Platalea ajaja, vista caudal; (13) Eudocimus ruber, vista ventral; (14) Mesembrinibis cayennensis, vista ventral. (acl) Ângulo caudolateral, (acm) ângulo caudomedial, (Ap) ala paraesfenóide, (BA) basioccipital, (Ch) canal do nervo hipoglosso, (CO) côndilo occipital, (CoC) côndilo caudal, (CoL) côndilo lateral, (CoM) côndilo medial, (CoM) côndilo medial, (CPt) côndilo pterigóide, $(\mathrm{Cr} 5)$ crista 5, (CrBt) crista basilar transversa, (CrL) crista lateral do palatino, (CrNt) crista nucal transversa, (CrT) crista temporal, (CrV) crista ventral do palatino, (EX) exoccipital, (F1) fossa 1, (F2) fossa 2, (Fc) fossa coanal, (fen) fenestra, (fo) fontículo occipital, (fo2) forâmen 2, (fog) forâmen do nervo glossofaringeal, (FoM) forâmen magno, (fov) forâmen do nervo vago, (Fp) fossa parabasal, (fsc) fossa subcondilar, (FV) fossa ventral, (J) arco jugal, (L) lacrimal, (LP) lâmina paraesfenóide, (Occ) óstio canal carótico, (Ocoe) óstio canal oftálmico externo, (P) parietal, (PAL) palatino, (PC) proeminência cerebelar, (PjP) projeção do paraesfenóide, (PM) premaxilar, (Pr10) processo 10, (Pr3-Pr9) processo 3 a (Pr9) processo 9, (Prj) prcesso jugal, (PrlP) processo lateral do paraesfenóide, (PrM) processo maxilar do palatino, (PrOt) processo ótico, (PrP) processo paraesfenóide, (PrP) processo paraoccipital, (PT) pterigóide, (RoP) rostro do paraesfenóide, (SO) supraoccipital, (Sul 1) sulco 1, (Sul 3) sulco 3, (tac) tuba auditiva comum, (Tb) tubérculo basilar, (V) vômer. 

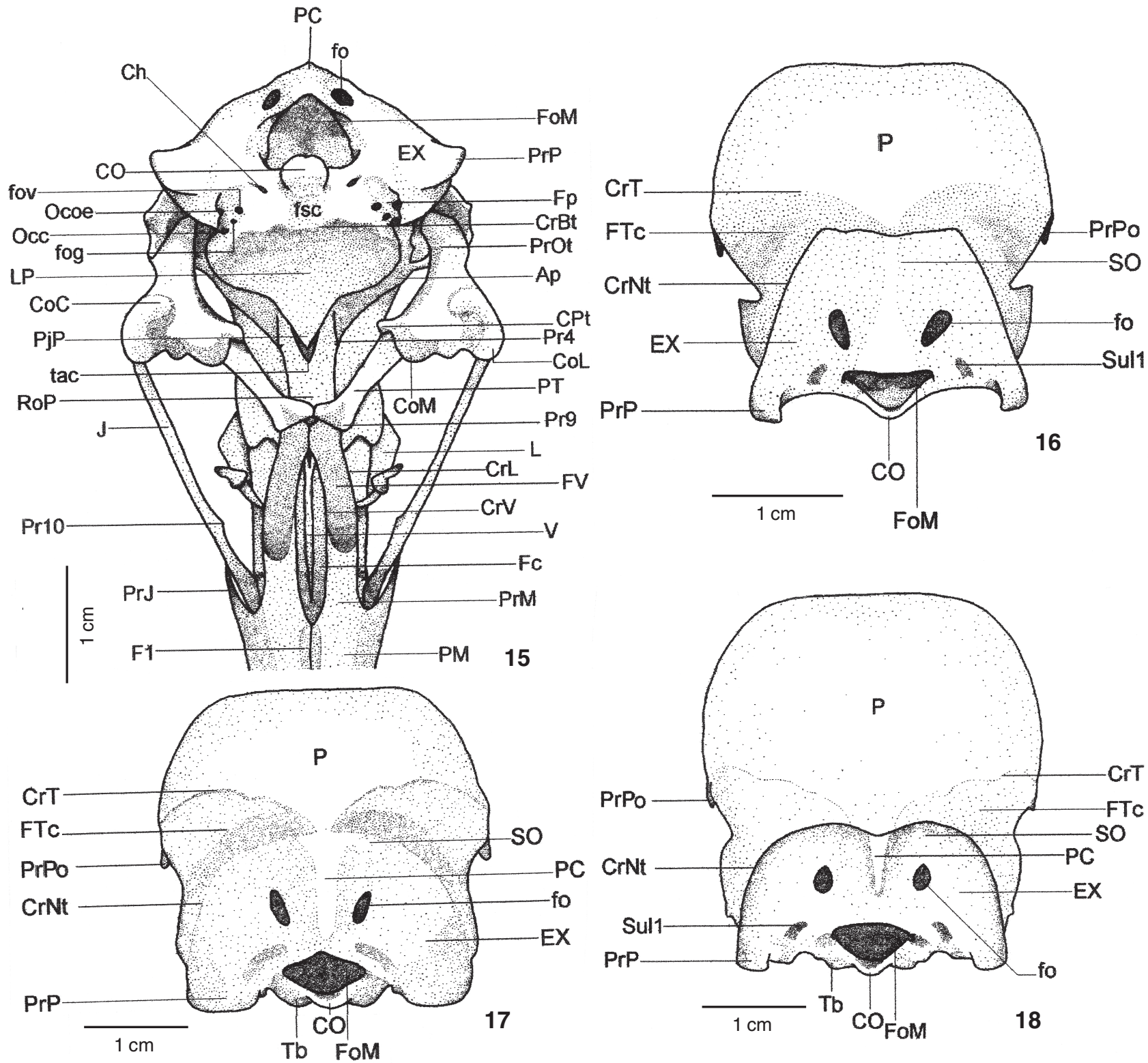

Figuras 15-18. Crânio. (15) Theristicus caudatus, vista ventral; (16-18) vista caudal: (16) Eudocimus ruber; (17) Mesembrinibis cayennensis; (18) Theristicus caudatus. (Ap) Ala paraesfenóide, (Ch) canal do nervo hipoglosso, (CO) côndilo occipital, (CoC) côndilo caudal, (CoL) côndilo lateral, $(\mathrm{CoM})$ côndilo medial, $(\mathrm{CPt})$ côndilo pterigóide, $(\mathrm{CrBt})$ crista basilar transversa, $(\mathrm{CrL}) \mathrm{crista}$ lateral do palatino, $(\mathrm{CrNt})$ crista nucal transversa, (CrT) crista temporal, (CrV) crista ventral do palatino, (EX) exoccipital, (F1) fossa 1, (Fc) fossa coanal, (fo) fontículo occipital, (fog) forâmen do nervo glossofaringeal, (FoM) forâmen magno, (fov) forâmen do nervo vago, (Fp) fossa parabasal, (fsc) fossa subcondilar, (FTc) fossa temporal caudal, (FV) fossa ventral, (J) arco jugal, (L) lacrimal, (LP) lâmina paraesfenóide, (Occ) óstio canal carótico, (Ocoe) óstio canal oftálmico externo, (P) parietal, (PC) proeminência cerebelar, (PjP) projeção do paraesfenóide, (PM) premaxilar, (Pr4) processo 4, (Pr9) processo 9, (Prj) processo jugal, (PrM) processo maxilar do palatino, (PrOt) processo ótico, (PrP) processo paraesfenóide, (PrP) processo paraoccipital, (PrPo) processo pós-orbital, (PT) pterigóide, (RoP) rostro do paraesfenóide, (SO) supraoccipital, (SO) supraoccipital, (Sul 1) sulco 1, (tac) tuba auditiva comum, (Tb) tubérculo basilar, (V) vômer.

Revista Brasileira de Zoologia 22 (3): 529-551, setembro 2005 

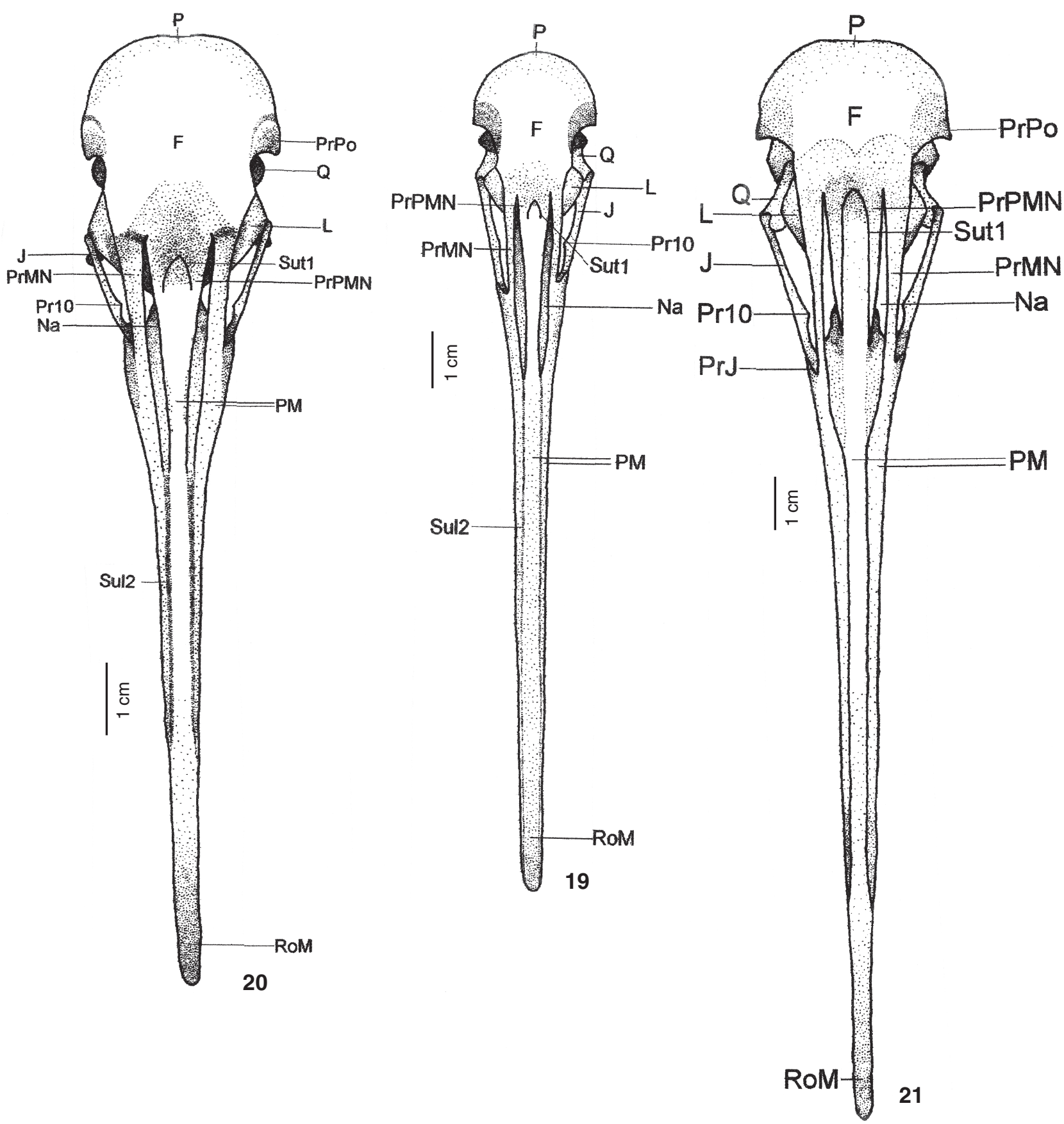

Figuras 19-21. Vista dorsal do crânio: (19) Eudocimus ruber; (20) Mesembrinibis cayennensis; (21) Theristicus caudatus. (F) Frontal, (J) arco jugal, (L) lacrimal, (Na) narina, (P) parietal, (PM) premaxilar, (Pr10) processo 10, (Prl) processo jugal, (PrMN) processo maxilar do nasal, (PrPMN) processo premaxilar do nasal, (PrPo) processo pós-orbital, (Q) quadrado, (RoM) rostro maxilar, (Sul 2) sulco 2, (Sut 1 ) sutura 1.

rostralmente; b) Processo suprameático (proc. suprameaticus, PrSm - Fig. 3), curto e afilado na sua extremidade, com cerca de 1/3 do comprimento do processo ótico do quadrado (proc. otici, PrOt - Fig. 3), forma parte da cótila quadrática esquamosal (cotyla quadratica squamosi) que se articula com o capítulo esquamoso (capitulum squamosum) do osso quadrado (BAUMEL \& WiTMER 1993); o processo suprameático contribui também para formar a borda dorsal do meato acústico externo (mae - 


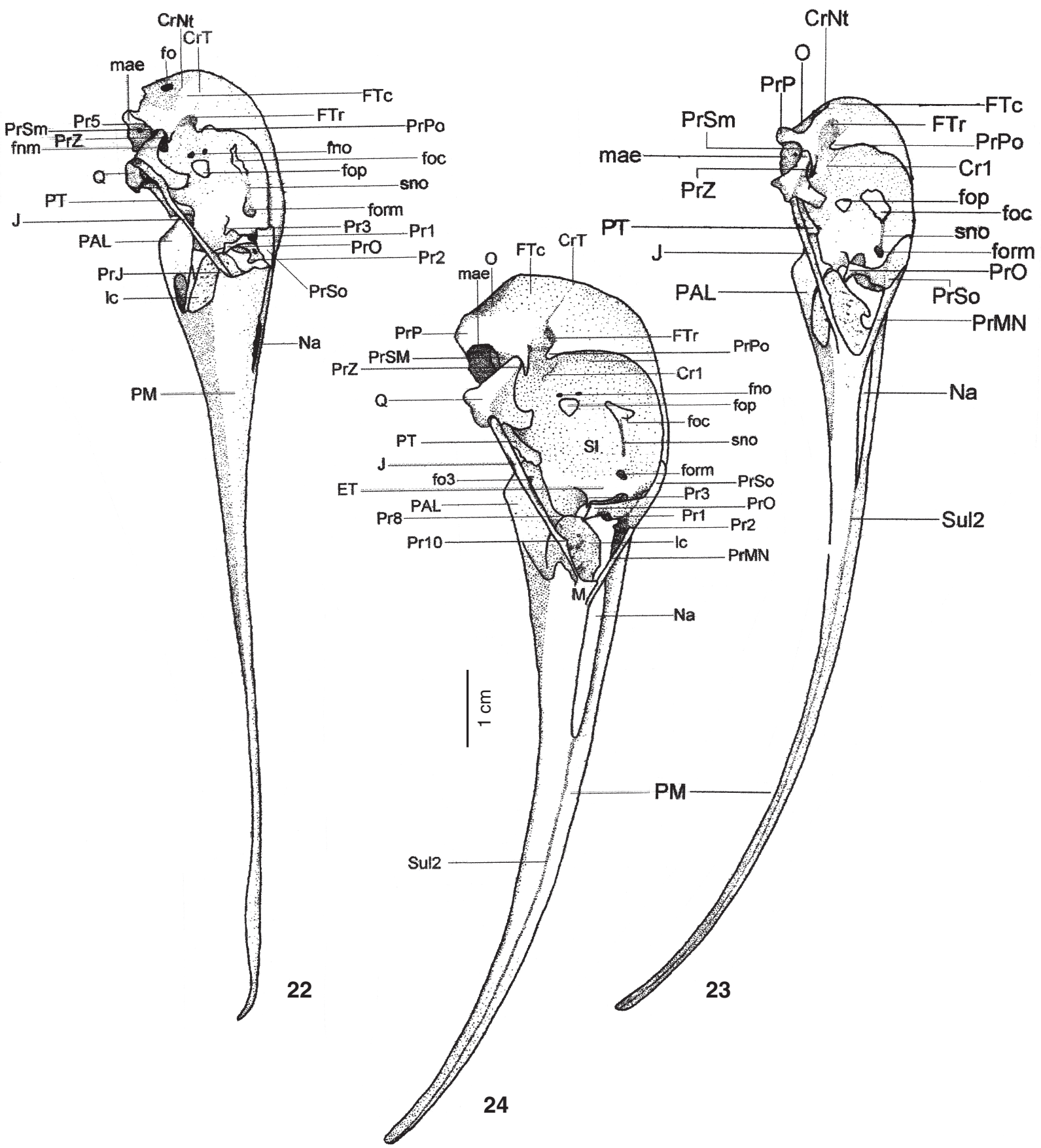

Figuras 22-24. Vista lateral do crânio: (22). Platalea ajaja; (23) Eudocimus ruber; (24) Mesembrinibis cayennensis. (Cr1) Crista 1, (CrNt) crista nucal transversa, (CrT) crista temporal, (ET) ectetmóide, (fnm) forâmen do nervo maxilomandibular, (fno) forâmen do nervo oftálmico, (fo) fontículo occipital, (fo3) forâmen 3, (foc) fontículo orbitocranial, (fop) forâmen óptico, (form) forâmen orbitonasal medial, (FTc) fossa temporal caudal, (FTr) fossa temporal rostral, (J) arco jugal, (Ic) lamela coanal, (mae) meato acústico externo, (Na) narina, (O) occipital, (PAL) palatino, (PM) premaxilar, (Pr1) processo 1, (Pr2) processo 2, (Pr3) processo 3, (Pr5) processo 5, (Pr8) processo 8, (Pr10) processo 10, (Prj) processo jugal do maxilar, (PrMN) processo maxilar do nasal, (PrO) processo orbital, (PrP) processo paraoccipital, (PrPo) processo pós-orbital, (PrSm) processo suprameático, (PrSo) proceso supra-orbital, (PrZ) processo zigomático, (PT) pterigóide, (Q) quadrado, (SI) septo interorbital, (sno) sulco do nervo olfatório, (Sul 2) sulco 2. 
Fig. 3); c) Processo 5 (Pr5 - Fig. 3), curto, localizado caudalmente ao processo suprameático.

O processo zigomático é mais desenvolvido em E. ruber (PrZ - Fig. 7) e apresenta-se achatado dorsoventralmente, medindo cerca de $1 / 3$ da distância de sua origem até o arco jugal; atinge seu máximo desenvolvimento em M. cayennensis (PrZ Fig. 8) e Threristicus caudatus (PrZ - Fig. 9), com pouco mais da metade desta distância, sendo mais robusto em Threristicus caudatus (PrZ - Fig. 9).

Nos Ardeidae estudados, este processo é pouco desenvolvido, podendo ainda ser reduzido em A. cocoi; em N. nycticorax, ele é achatado dorsoventralmente. É pouco desenvolvido também em $P$. ruber, sendo desenvolvido em C. atratus. Em Ciconiidae é muito bem desenvolvido, sendo que em C. maguari é achatado dorsoventralmente em sua porção proximal e laterolateralmente, em sua porção distal; já em M. americana o processo zigomático é achatado dorsoventralmente e afila-se lateralmente, formando um processo que se orienta ventralmente; $J$. mycteria apresenta este processo achatado dorsoventralmente na sua porção mais distal e se orienta rostroventralmente.

De acordo com VerheYen (1959), este processo é melhor desenvolvido em Threskiornithidae e Ciconiidae que em Ardeidae, Scopidae e Balaenicipitidae, sendo vestigial nos Phoenicopteridae, o que corrobora com o observado no presente estudo.

Não se observa variação do processo suprameático nos Threskiornithidae (PrSm - Figs 3, 7-9); contudo, é pouco desenvolvido em A. cocoi, A. alba, B. striatus, E. thula e N. nycticorax, sendo curto em T. lineatum. Em P. ruber é bem desenvolvido, assim como C. maguari, M. americana e J. mycteria; está ausente em C. atratus.

O processo 5 do esquamosal é mais desenvolvido e largo na base em M. cayennensis (Pr5 - Fig. 8), seguido por Threristicus caudatus (Pr5 - Fig. 9) e P. ajaja (Pr5 - Fig. 3), onde se mostra curto; está ausente em $E$. ruber, bem como nas demais espécies estudadas.

A fossa temporal (fossa temporalis) de $P$. ajaja está subdividida em duas partes: a) Fossa temporal rostral (FTr - Fig. 3) que é curta, profunda e delimitada rostralmente pela crista 1 (Cr1 Fig. 3) e caudalmente pela crista 2 ( $\mathrm{Cr} 2$ - Fig. 3); e b) Fossa temporal caudal (FTc - Fig. 3) que é rasa, mais comprida que larga e limitada rostralmente pela crista 2 (Cr2 - Fig. 3), dorsalmente pela crista temporal (CrT - Fig. 3) e caudalmente pela crista nucal transversa ( $\mathrm{CrNt}$ - Fig. 3 ).

Nota-se que a fossa temporal é bem desenvolvida, de uma forma geral, nos Threskiornithidae estudados. A fossa temporal rostral é mais larga em E. ruber (FTr - Fig. 7), seguido por Threristicus caudatus (FTr - Fig. 9), P. ajaja (FTr - Fig. 3) e M. cayennensis (FTr - Fig. 8), onde se mostra menos desenvolvida. Esta fossa é bem desenvolvida nos Ardeidae, sendo que nos Ciconiidae é bem profunda. Já a fossa temporal caudal é desenvolvida em todos os Threskiornithidae estudados, atingindo seu máximo de desenvolvimento em M. cayennensis (FTc - Fig. 8), seguido por E. ruber (FTc - Fig. 7), Threristicus caudatus (FTc
- Fig. 9) e P. ajaja (FTc - Fig. 3); os Ardeidae também possuem a fossa temporal caudal bem desenvolvida; já nos Ciconiidae mostra-se mais rasa. Em C. atratus é uma fossa temporal única, não estando dividida em rostral e caudal como nas outras espécies estudadas, e não se observam as cristas 1 e 2 .

Contudo, segundo VerheYen (1959), a fossa temporal é curta e geralmente mal delimitada nos Threskiornithidae (salvo Ibis), o que não foi observado no presente estudo. VERHEYEN (1959) ainda relata que em Ciconiidae (salvo Mycteria), Balaenicipitidae, Scopidae e Phoenicopteridae, a fossa temporal também é curta e mal delimitada, sendo larga e profunda nos Ardeidae.

Segundo Donatelli (1997), o desenvolvimento da fossa temporal pode estar associado com o desenvolvimento dos músculos adutores mandibulares externos. Assim, quanto maior o desenvolvimento destes músculos, maior mostra-se a fossa temporal. Para Pycraft (1903) o tamanho e profundidade da região esquamosal depende fortemente do desenvolvimento da fossa temporal.

A crista temporal é conspícua entre os Treskiornithidae estudados, sendo que em Threristicus caudatus (CrT - Figs 9 e 18), E. ruber (CrT - Figs 7 e 16) e M. cayennensis (CrT - Figs 8 e 17) assemelha-se a de P. ajaja (CrT - Figs 3 e 12), na qual é bem mais conspícua. É muito bem desenvolvida em $A$. cocoi, $T$. lineatum e $A$. alba, estando ausente em $P$. ruber. Já a crista nucal transversa (CrNt - Figs 12, 16-18) está presente em todas as espécies estudadas, sendo menos desenvolvida em $T$. caudatus (CrNt - Fig. 18), A. cocoi, T. lineatum e A. alba, seguidos por $B$. striatus, E. thula, N. nycticorax e M. americana.

A região do lateroesfenóide (LA - Fig. 3) está limitada com o osso esquamosal (os squamosum, E - Fig. 3) por meio da crista 1 (Cr1 - Fig. 3).Nesta região observa-se um pequeno processo 6 em Threristicus caudatus (Pr6 - Fig. 9), E. ruber (Pr6 - Fig. 7) e M. cayennensis (Pr6 - Fig. 8), que se localiza caudoventralmente e orienta-se ventrorrostralmente, mas não é observado em P. ajaja. Este processo está presente somente nos Threskiornithinae e Ciconiidae estudados, e não foi relatado por nenhum outro autor.

O forâmen do nervo oftálmico (foramen nervus ophthalmici, fno) é conspícuo em P. ajaja (fno - Fig. 3) e localiza-se na porção ventrolateral do lateroesfenóide. Ventralmente, observa-se o forâmen do nervo maxilomandibular (foramen nervus maxillomandibularis, fnm - Fig. 3) bem desenvolvido.

P. ajaja e Threristicus caudatus apresentam o forâmen do nervo oftálmico (fno - Figs 3 e 9) mais conspícuo que em $E$. ruber (fno - Fig. 7) e M. cayennensis (fno - Fig. 8). Nos Ardeidae, este forâmen funde-se ao forâmen óptico; já em M. americana, J. mycteria e C. atratus este é pouco conspícuo.

O forâmen do nervo maxilomandibular é conspícuo em P. ajaja (fnm - Fig. 3) e E. ruber (fnm - Fig. 7), seguidos por Threristicus caudatus (fnm - Fig. 9) e M. cayennensis (fnm - Fig. 8); em A. cocoi, T. lineatum, A. alba, E. thula, N. nycticorax, C. maguari, M. americana e J. mycteria este forâmen é menos conspícuo.

Revista Brasileira de Zoologia 22 (3): 529-551, setembro 2005 
Observa-se também na região do lateroesfenóide, o processo pós-orbital (proc. postorbitalis, PrPo) que é curto em P. ajaja (PrPo - Fig. 3), mas conspícuo e orienta-se rostroventralmente; apresenta cerca de $1 / 5$ da distância de sua origem na caixa craniana até o arco jugal.

O processo pós-orbital é mais desenvolvido em Threristicus caudatus (PrPo - Fig. 9) com cerca de 1/3 do distância de sua origem na caixa craniana até o arco jugal; E. ruber (PrPo - Fig. 7) e M. cayennensis (PrPo - Fig. 8) apresentam o peocesso pósorbital um pouco menos desenvolvido, cerca de $1 / 4$ desta distância. Este processo é muito bem desenvolvido em J. mycteria, C. maguari, M. americana, C. atratus, P. ruber, E. thula e B. striatus; já em A. cocoi, T. lineatum e A. alba o processo pós-orbital é pouco desenvolvido. Em $N$. nycticorax, ele é mais estreito que nas demais espécies.

Os Threskiornithidae apresentam o processo pós-orbital bem desenvolvido, principalmente nos Threskiornithinae, fato este que não corrobora com as observações de VERHEYEN (1959). Este autor relata que o processo pós-orbital é ausente ou vestigial entre os Threskiornithidae e Ciconiidae, ausente nos Phoenicopteridae e Scopidae e pouco desenvolvido nos Ardeidae, com exceção de Cochlearius onde se apresenta desenvolvido, mas curto.

\section{Os basioccipitale, os exoccipitale, os supraoccipitale}

A região do osso supra-occipital (SO - Fig. 12) de P. ajaja limita-se dorsalmente com o osso parietal (P - Fig. 12) por meio da crista nucal transversa (CrNt - Fig. 12); lateralmente fusionase com o osso exoccipital (EX - Fig. 12) e ventralmente participa na formação da borda dorsal do forâmen magno (foramen magnum, FoM - Fig. 12).

Na sua porção mediana, observa-se uma típica proeminência cerebelar (proeminentia cerebellaris, PC - Fig. 11). Lateralmente a esta proeminência, constata-se um par de fontículos occipitais (fonticulus occipitalis, fo - Fig. 12) bem desenvolvidos e conspícuos.

A proeminência cerebelar é saliente em Threristicus caudatus (PC - Fig. 15), seguido por E. ruber (PC - Fig. 13) e M. cayennensis (PC - Fig. 14), sendo menos evidente em $P$. ajaja (PC - Fig. 11). Nas demais espécies estudadas, é também saliente em $B$. striatus, E. thula, C. maguari, M. americana, J. mycteria e C. atratus e muito saliente em $P$. ruber; é reduzida em Tigrisoma linetum e A. alba, estando ausente ou muito reduzida em A. cocoi.

VERHEYEN (1959), relata que a proeminência cerebelar é bem desenvolvida em Phoenicopteridae e Threskiornithidae, mas é praticamente ausente entre os Ardeidae, Scopidae, Balaenicepdae e Ciconiidae (exceto Anastomus $e$ Sphenorhynchus). Contudo, no presente estudo observou-se que a proeminência cerebelar é desenvolvida em C. maguari, Jabiru mycteria e M. americana (Ciconiidae), bem como em B. striatus e E. thula (Ardeidae), quando comparada aos Threskiornithidae.

Lateroventralmente ao forâmen magno, pode-se observar o forâmen da veia occipital externa (foramen venum occipitalis externae) localizado num sulco (Sul 1 - Fig. 12). Tal forâmen foi observado em dois dos quatro indivíduos de Threristicus caudatus estudados, estando presente nas demais espécies estudadas.

Em P. ajaja, a região do exoccipital (EX - Fig. 12) limitase com a porção caudal do meato acústico externo por meio do desenvolvido e conspícuo processo paraoccipital (proc. paraoccipitalis, PrP - Fig. 12), que se projeta lateroventralmente e possui cerca da metade da extensão do processo ótico (PrOt - Fig. 3) do osso quadrado.

O processo paraoccipital é bem desenvolvido nos Threskiornithidae, possui cerca da metade da extensão do processo ótico do osso quadrado em P. ajaja (PrP - Figs 11 e 12), quase a metade desta extensão em Threristicus caudatus (PrP - Figs 15 e 18) e M. cayennensis (PrP - Figs 14 e 17). Em E. ruber (PrP - Figs 13 e 16), este processo atinge o seu máximo desenvolvimento, cerca de $2 / 3$ da extensão do processo ótico do osso quadrado. Apresenta-se curto nos Ardeidae, sendo que em A. alba, B. striatus e $E$. thula orienta-se ventralmente; este processo é bem reduzido em N. nycticorax. Já M. americana e J. mycteria é curto e orienta-se ventrorrostralmente; em $C$. atratus este processo orienta-se ventralmente, em $P$. ruber projeta-se ventromedialmente.

A fossa parabasal (fossa parabasalis, Fp - Fig. 11) localizase ventrorrostralmente na região do osso exoccipital e medialmente ao meato acústico externo (mae - Fig. 3). Tal fossa é profunda e abriga quatro aberturas: Forâmen do nervo vago (foramen nervus vagi, fov - Fig. 11), localizado caudomedialmente na fossa; óstio canal oftálmico externo (ostium canalis ophthalmici externi, Ocoe - Fig. 11), caudolateralmente na fossa, paralelo ao anterior; forâmen do nervo glossofaringeal (foramen nervus glossopharyngealis, fog - Fig. 11), rostralmente ao forâmen nervo vago; e óstio canal carótico (ostium canallis carotici, Occ - Fig. 11), localizado bem rostralmente na fossa.

Ainda na região exoccipital, observam-se duas aberturas (canalis nervus hipoglossi, Ch - Fig. 11) localizadas lateralmente ao côndilo occipital (CO - Figs 11 e 12).

A região do osso basioccipital (BA - Fig. 11) de P. ajaja encontra-se fusionada lateralmente com o osso exoccipital (EX - Fig. 11); rostralmente contata-se com o osso paraesfenóide por meio da crista basilar transversa (CrBt - Fig. 11), onde se observa os conspícuos tubérculos basilares (Tb - Fig. 12).

Nota-se que o tubérculo basilar é pouco desenvolvido em $A$. cocoi e T. lineatum, reduzido em A. alba e ausente em B. striatus, E. thula e N. nycticorax. Apresenta-se excepcionalmente saliente em $P$. ruber e conspícuo em C. maguari.

Em sua porção caudal, a região basioccipital contata-se com o bem desenvolvido côndilo occipital (CO - Fig. 11 e 12), que se projeta ventrocaudalmente; observa-se rostralmente a este, a fossa subcondilar (fossa subcondylaris, fsc - Fig. 11) bem definida, profunda e conspícua.

A fossa subcondilar é mais profunda em Threristicus caudatus (fsc - Fig. 15) que nos demais Threkiornithidae (fsc - Figs 11,13 e 14) estudados; esta fossa é, ainda, profunda em $C$. atratus e $P$. ruber; rasa em T. lineatum, A. alba e N. nycticorax e ausente em A. cocoi, B. striatus e E. thula. 


\section{Ossa faciei \\ Os nasale, os premaxillare, os maxillare}

A maxila superior corresponde a quatro vezes o tamanho da caixa craniana em P. ajaja.

O osso nasal (N - Figs 1 e 2) articula-se caudalmente com o osso frontal (F - Figs 1 e 2) na zona flexória craniofacial (zona flexoria craniofacialis); contata-se com o osso lacrimal (L - Figs 1 e 2) por meio da sutura lacrimofrontal (slf - Fig. 2); lateralmente fusiona-se com o osso maxilar e medialmente contatase com o osso premaxilar (PM - Figs 1 e 2) por meio de uma sutura (Sut 1 - Fig. 1 e 2).

Nos Threskiornithinae, o osso nasal contata-se rostrolateralmente com o osso maxilar por meio do processo maxilar (PrMN - Figs 4-6, 19, 21 e 26) e medialmente contata-se com o osso premaxilar por meio do processo premaxilar (PrPMN Figs 4-6, 19, 20 e 21).

Em P. ajaja, as suturas 1 (Sut1 - Figs 1 e 2) apresentam-se paralelamente uma a outra, enquanto que nos Threskiornithinae (Sut1 - Figs 4-6, 19, 20 e 21) unem-se caudalmente, sendo pouco visível dependendo do espécimen. Estas são pouco conspícuas em A. cocoi, estando ausentes em A. alba, E. thula, N. nycticorax, M. americana e J. mycteria.

A narina (apertura nasi ossea, Na - Figs 1 e 2) de P. ajaja localiza-se caudalmente na maxila superior, apresenta forma elíptica e é mais rasa rostralmente e profunda caudalmente. Entre os Threskiornithidae, a narina localizada-se em um sulco (Sul 2 - Figs 1, 23-25) que se estende até a extremidade do bico. Apresenta-se estreita e longa nos Threskiornithinae ( $\mathrm{Na}$ - Figs 19-21) e em P. ajaja (Na - Fig. 1), é curta e tem forma ovalada. É do tipo esquizorrina entre os Threskiornithinae, diferentemente de $P$. ajaja (Plataleinae) que é do tipo pseudo-holorrina; nos Ciconiidae, Ardeidae e Phoenicopteridae observa-se o tipo holorrina (VERHEYEN 1959).

Segundo Verheyen (1959), as narinas dos Ciconiiformes são alongadas e grandes entre os Ardeidae (com exceção de Cochlearius), assim como entre os Threskiornithidae e os Phoenicopteridae; nos Threskiornithidae, a narina localiza-se em um sulco. Todavia, dentre os Threskiornithidae estudados neste trabalho, observou-se que nos Threskiornithinae as narinas são alongadas e estreitas enquanto nos Plataleinae, estas são curtas e mais largas.

Em P. ajaja o osso premaxilar (PM - Figs 1 e 2) contata-se caudolateralmente com o osso nasal por meio da sutura 1 (Sut1 - Figs 1 e 2), separando assim os ossos nasais entre si; caudalmente contata-se com o osso frontal, definindo a zona flexora craniofacial e laterocaudalmente funde-se com o osso maxilar. Um sulco (Sul 2 - Fig. 1) característico é observado na sua face dorsal.

O osso premaxilar é um osso estreito e curvado em $E$. ruber (PM - Figs 19 e 23), M. cayennensis (PM - Figs 20 e 24) e Threristicus caudatus (PM - Figs 21 e 25), uma característica que separa os Threskiornithinae dos Plataleinae (PM - Fig. 22). Todas as espécies de Threskiornithidae apresentam pequenos foramens (foramina neurovascularia) nas extremidades das superfícies dorsal e ventral do premaxilar; estes foramens também estão presentes em $P$. ruber. Tal fato corrobora com o observado por VERHEYEN (1959), que relata que o premaxilar é estreito nos Threskiornithinae, Ardeidae, Ciconiidae, com exceção dos Plataleinae e Phoenicopteridae. Nos Ciconiiformes, este osso termina distalmente em uma ponta afilada reta ou curvada, com exceção de Balaeniceps (Balaenicipitidae), onde a ponta da maxila superior é constituída por um gancho robusto e em Plataleinae, é arredondada.

O rostro maxilar (rostrum maxillae) é achatado dorsoventralmente em P. ajaja (RoM - Fig. 1), levemente curvado e largo em sua extremidade rostral, tomando forma característica de uma colher. É perfurado por pequenos forâmens (foramina neurovascularia) nas suas superfícies dorsal e ventral. Ventralmente, o osso premaxilar ocupa grande parte da maxila superior e fusiona-se ventrocaudalmente com o palatino (PAL - Fig. $10)$.

Observa-se um sulco 3 (Sul 3 - Fig. 10) característico na porção mediana da face ventral do osso premaxilar de $P$. ajaja; caudalmente, este sulco contata-se com uma fossa estreita (F1

- Fig. 10), que apresenta duas aberturas (canalis neurovascularis maxillae, cnm - Fig. 10), e em alguns indivíduos, podem se apresentar fundidas. Esta fossa contata-se caudalmente com o vômer (V - Figs 10 e 11) e caudolateralmente com o osso palatino (PAL - Fig. 10).

O sulco 3 é bem desenvolvido nos Threskiornithidae (Sul 3 - Figs 10, 26-28); é profundo nos Ciconiidae estudados, delimitado por uma crista, mas não atinge a porção caudal da mandíbula. Em Ardeidae, encontra-se uma crista no lugar do sulco; não se observa tais estruturas em $C$. atratus e $P$. ruber.

M. cayennensis e Threristicus caudatus possuem uma fenestra (fen - Figs 27 e 28) localizada rostralmente na fossa 1 (F1 - Figs 27 e 28) em vez das duas aberturas observadas em $P$. ajaja (cnm - Fig. 10) e E. ruber (cnm - Fig. 26); tal estrutura pode variar de tamanho, podendo ainda ser pouco profunda. Não se observa esta fossa (F1) nos Ardeidae, bem como em $C$. atratus; já em C. maguari, M. americana e J. mycteria esta fossa é rasa com pequenos foramens.

Ainda na face ventral do premaxilar, há uma fossa rasa (F2 - Figs 10 e 11) localizada caudolateralmente. Pode-se ainda observar um forâmen (fo2 - Figs 10 e 11) localizado na sua porção rostral. Estas estruturas estão presentes somente em $P$. ajaja.

A região do maxilar é reduzida nos Threskiornithidae e funde-se ao osso premaxilar. Tal região articula-se caudalmente com o arco jugal por meio do processo jugal (proc. jugalis, PrJ, Figs 3 e 10) do osso maxilar.

\section{Os palatinum, vomer, os pterygoideum, os jugale}

O palato dos Threskiornithidae é do tipo desmognato, segundo a classificação de Huxley (1867), onde o vômer quando presente é estreito e afilado e o maxilopalatino une-se medianamente e torna-se largo e esponjoso, ocupando a base da maxila superior. 


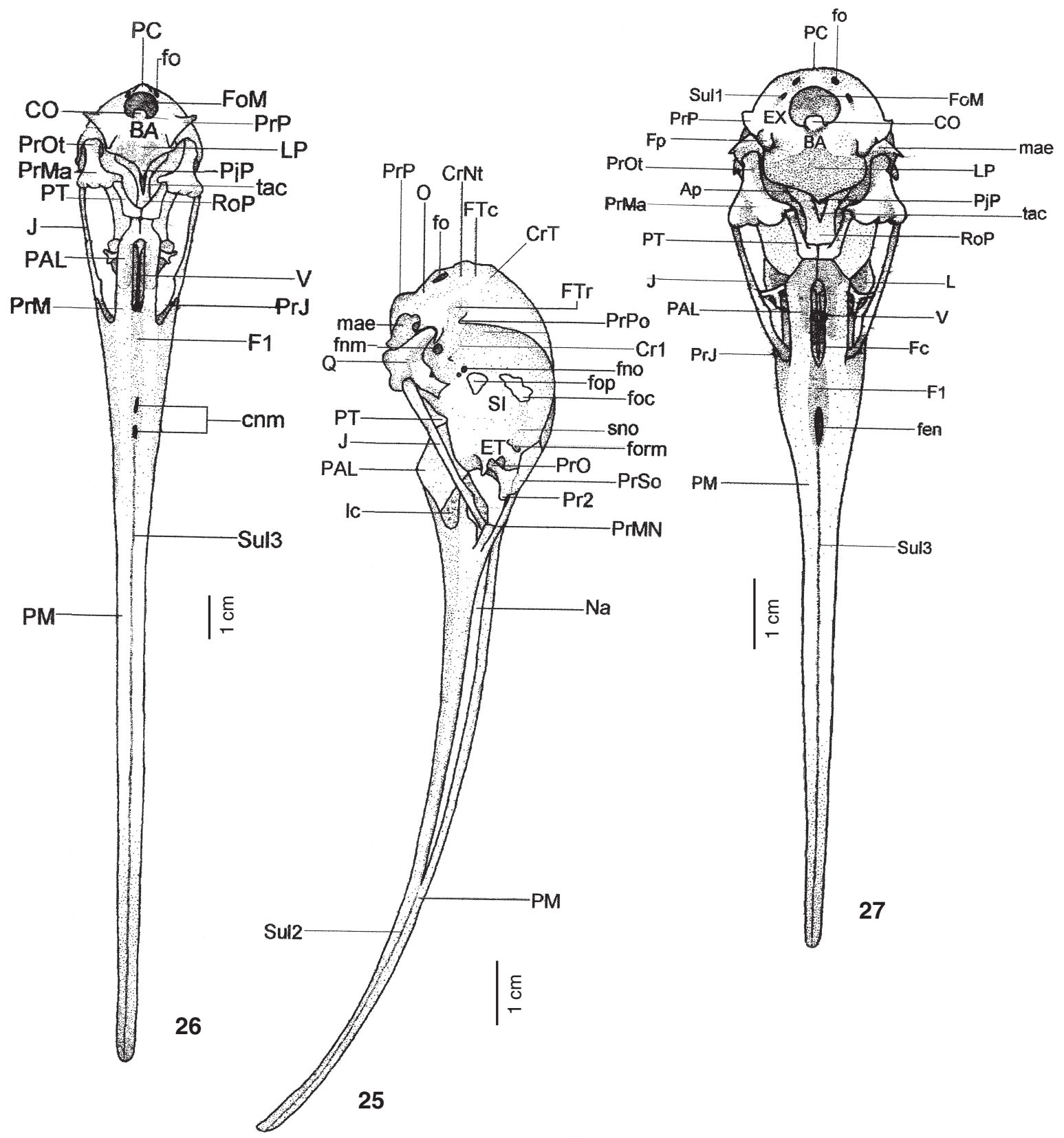

Figuras 25-27. Crânio. (25) Vista lateral: Theristicus caudatus; (26-27) vista ventral: (26) Eudocimus ruber; (27) Mesembrinibis cayennensis. (Ap) Ala paraesfenóide, (BA) basioccipital, (cnm) canal neurovascular da maxila, (CO) côndilo occipital, (Cr1) crista 1, (CrNt) crista nucal transversa, (CrT) crista temporal, (ET) ectetmóide, (EX) exoccipital, (F1) fossa 1, (Fc) fossa coanal, (fen) fenestra, (fnm) forâmen do nervo maxilomandibular, (fno) forâmen do nervo oftálmico, (fo) fontículo occipital, (foc) fontículo orbitocranial, (FoM) forâmen magno, (fop) forâmen óptico, (form) forâmen orbitonasal medial, (Fp) fossa parabasal, (FTc) fossa temporal caudal, (FTr) fossa temporal rostral, (J) arco jugal, (L) lacrimal, (Ic) lamela coanal, (LP) lâmina paraesfenóide, (mae) meato acústico externo, ( $\mathrm{Na}$ ) narina, (O) occipital, (PAL) palatino, (PC) proeminência cerebelar, (PjP) projeção do paraesfenóide, (PM) premaxilar, (Pr2) processo 2, (Prj) prcesso jugal, (PrM) processo maxilar do palatino, (PrMa) processo mandibular, (PrMN) processo maxilar do nasal, (PrO) processo orbital, (PrOt) processo ótico, (PrP) processo paraesfenóide, (PrP) processo paraoccipital, (PrPo) processo pós-orbital, (PrSo) proceso supra-orbital, (PT) pterigóide, (Q) quadrado, (RoP) rostro do paraesfenóide, (SI) septo interorbital, (sno) sulco do nervo olfatório, (Sul 1) sulco 1, (Sul 2) sulco 2, (Sul 3) sulco 3, (tac) tuba auditiva comum, (V) vômer.

Revista Brasileira de Zoologia 22 (3): 529-551, setembro 2005 


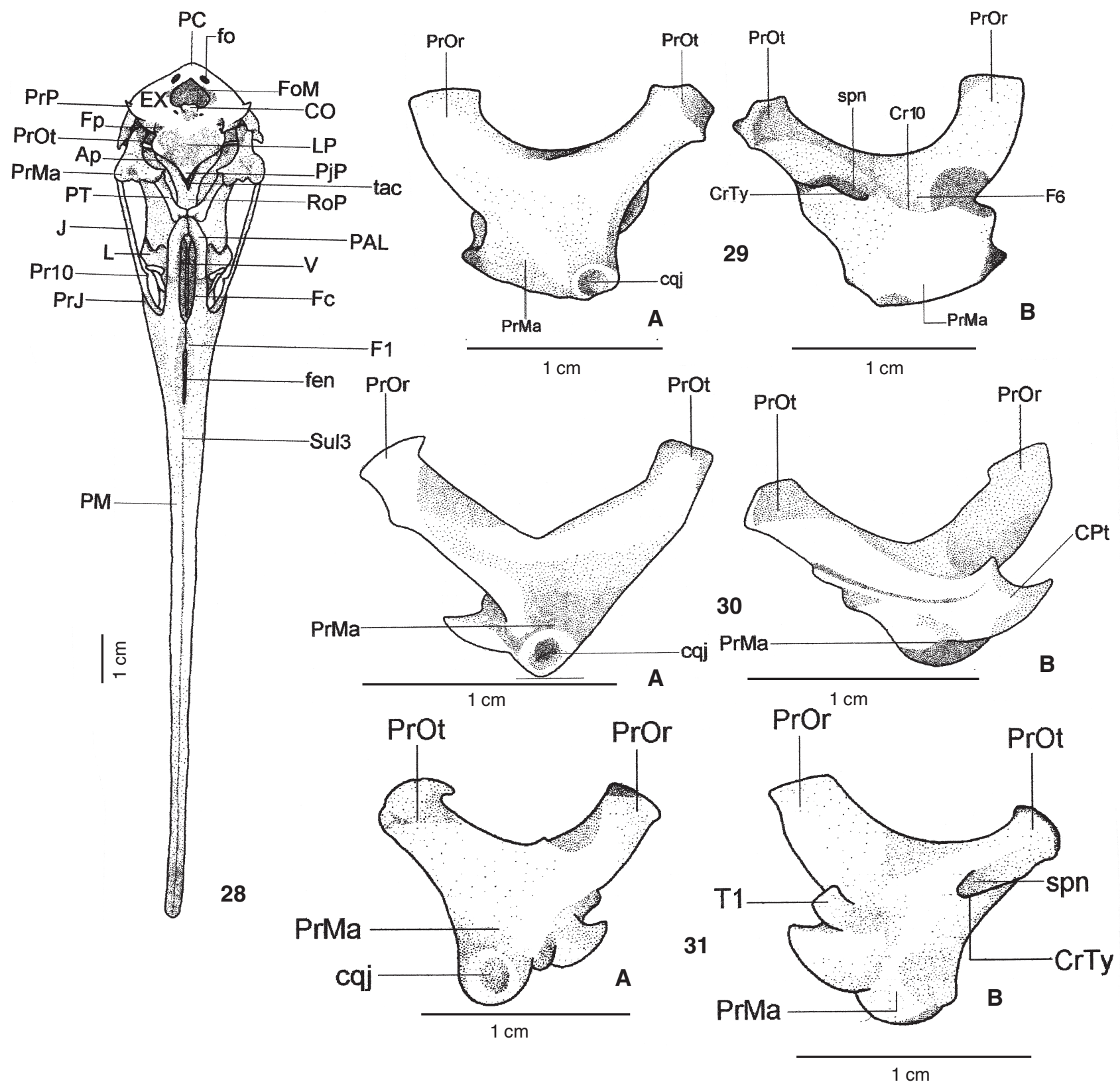

Figuras 28-31. 28. Theristicus caudatus: vista ventral do crânio; (29) Platalea ajaja, quadrado: (A) vista lateral, (B) vista medial; (30) Eudocimus ruber, quadrado: (A) vista lateral, (B) vista medial; (31) Theristicus caudatus, quadrado: (A) vista lateral, (B) vista medial. (Ap) Ala paraesfenóide, (BA) basioccipital, (cnm) canal neurovascular da maxila, (CO) côndilo occipital, (CPt) côndilo pterigóide, (Cqj) cótila quadradojugal, (Cr10) crista 10, (CrTy) crista timpânica, (EX) exoccipital, (F1) fossa 1, (F6) fossa 6, (Fc) fossa coanal, (fen) fenestra, (fo) fontículo occipital, (FoM) forâmen magno, (Fp) fossa parabasal, (J) arco jugal, (L) lacrimal, (LP) lâmina paraesfenóide, (PAL) palatino, (PC) proeminência cerebelar, (PjP) projeção do paraesfenóide, (PM) premaxilar, (Pr10) processo 10, (Prj) prcesso jugal, (PrM) processo maxilar do palatino, (PrMa) processo mandibular, (PrOr) processo orbital, (PrOt) processo ótico, (PrP) processo paraesfenóide, (PT) pterigóide, (RoP) rostro do paraesfenóide, (spn) sulco pneumático, (Sul 3) sulco 3, (T1) tubérculo 1, (tac) tuba auditiva comum, (V) vômer. 
Os Threskiornithidae apresentam o palatino na forma de duas barras paralelas onde se observa uma pequena variação entre as espécies estudadas. Já entre os Ardeidae (PAYNe \& Risley 1976), o formato do palatino varia de estreito, como em Ardea herodias, Ardeola striata virescens, Syrigma sibilatrix, Nyctanassa violacea e N. nycticorax a largo, em Cochlearius cochlearius.

A crista lateral (crista lateralis, CrL - Fig. 11) palatina de $P$. ajaja continua-se caudalmente com o ângulo caudolateral do palatino (angulus caudolateralis, acl - Fig. 11), com $45^{\circ}$ em relação à crista lateral; medialmente, observa-se a crista ventral do palatino (crista ventralis, CrV - Fig. 11). Caudomedialmente à crista ventral, observa-se o ângulo caudomedial (angulus caudomedialis, acm - Fig. 11) palatino e caudalmente à crista ventral, nota-se um curto e conspícuo processo 7 (Pr7 - Fig. 11).

A fossa ventral (fossa ventralis, FV - Figs 10 e 11) é delimitada pela crista lateral (CrL - Fig. 11) e pela crista ventral (CrV - Fig. 11). Tal fossa é profunda na sua porção caudal e rasa na sua porção rostral.

A crista lateral do osso palatino mostra-se bem desenvolvida em Threristicus caudatus (CrL - Fig. 15), atingindo um maior desenvolvimento em E. ruber (CrL - Fig. 13) e M. cayennensis (CrL - Fig. 14), assim como a crista ventral (CrV - Figs 13-15); em P. ajaja (CrL e CrV - Fig. 11), tais estruturas não se mostram tão desenvolvidas, bem como a fossa ventral que é rasa nesta espécie (FV - Fig. 11). A fossa ventral é mais profunda em $E$. ruber (FV - Fig. 13) e M. cayennensis (FV - Fig. 14) em relação à Threristicus caudatus (FV - Fig. 15). Nos Ardeidae e Ciconiidae estudados, a crista lateral é muito bem desenvolvida e a crista ventral segue praticamente o mesmo padrão.

O ângulo caudolateral não apresenta muita variação entre os Threskiornithidae (acl - Figs 11, 13 e 14), exceto em $T$. caudatus onde é bem reduzido; nos Ardeidae é afilado como um processo.

O processo 7 do palatino é curto e conspícuo em $P$. ajaja (Pr7 - Fig. 11), porém está ausente em E. ruber, M. cayennensis e em dois indivíduos de T. caudatus dos quatro analisados. Nos demais T. caudatus analisados, tal processo assemelha-se ao de $P$. ajaja. Nos Ardeidae, pode ser desenvolvido em $A$. cocoi, curto em T. lineatum e $N$. nycticorax e ausente em $A$. alba e B. striatus, bem como nos Ciconiidae. Este processo orienta-se caudalmente em P. ruber.

Observa-se que em P. ajaja a fossa coanal (fossa choanalis, Fc - Fig. 11) é profunda, longa e estreita e está delimitada pelas cristas ventrais ( $\mathrm{CrV}$ - Fig. 11). A parede medial do palatino é formada pela lamela coanal (lamella choanalis, lc - Fig. 3) bem desenvolvida, o que torna a fossa coanal profunda.

A face lateral do osso palatino de $P$. ajaja projeta-se dorsomedialmente e possui um processo 8 (Pr8 - Fig. 3) curto e conspícuo, localizado na sua porção dorsal. Tal processo é bem desenvolvido em E. ruber (Pr8 - Fig. 7), M. cayennensis (Pr8 - Fig. 8) e Threristicus caudatus (Pr8 - Fig. 9) e, nestas espécies, observase um forâmen (fo3 - Figs 7-9) localizado caudalmente nesta face. Em P. ajaja (Pr8 - Fig. 3), o processo 8 é curto e não se observa o forâmen (fo3) como nas espécies acima. Tal processo é reduzido em $A$. cocoi, pouco desenvolvido em T. lineatum e ausente em N. nycticorax; nos demais Ardeidae não há variação nesta estrutura, assemelhando-se ao de P. ajaja. Em C. maguari está reduzido e arredondado na extremidade, em $M$. americana e J. mycteria o mesmo orienta-se dorsocaudalmente, sendo afilado na sua extremidade; está ausente em $C$. atratus e $P$. ruber.

Na porção dorsorrostral do palatino de $P$. ajaja, há uma fossa (F3 - Fig. 3) que se estende da metade do osso palatino até a sua porção mais rostral. Esta fossa é profunda na sua porção caudal e rasa na sua porção rostral; está delimitada lateralmente pela crista 3 (Cr3 - Fig. 3) e medialmente pela crista 4 (Cr4 - Fig. 3).

A fossa F3 da porção dorsorrostral do palatino é menor em M. cayennensis (F3 - Fig. 8), Threristicus caudatus (F3 - Fig. 9) e E. ruber (F3 - Fig. 7) que a observada em P. ajaja (F3 - Fig. 3); está presente somente em N. nycticorax, estando ausente nas demais espécies.

O vômer (V - Figs 10 e 11) é achatado lateromedialmente e ventralmente, apresenta uma crista 5 em toda sua extensão, presente somente em $P$. ajaja (Cr5 - Fig. 11).

Nos Threskiornithidae o vômer é achatado lateromedialmente; nos Ciconiidae e $P$. ruber, o vômer também é achatado lateromedialmente, sendo que C. maguari tem um desenvolvido processo localizado ventrorrostralmente e que se orienta ventralmente; já em J. mycteria, funde-se rostralmente com a porção caudomedial do palatino. Nos Ardeidae, o vômer apresenta um sulco localizado medianamente e limitado lateralmente por cristas que se unem rostralmente. Tais constatações corroboram com as descrições de Verheyen (1959). Este autor relata que nos Threskiornithidae e Ciconiidae, o vômer tem, desde sua origem, o aspecto de estilete ou de lâmina, e entre Scopidae e Balaenicipitidae, possui a forma de uma curta lâmina triangular.

O osso pterigóide (PT - Figs 10 e 11) de P. ajaja é curto e, rostralmente, na região de articulação com o palatino, apresenta uma expansão lateral característica, formando um curto e arredondado processo 9 (Pr9 - Fig. 11). Tal processo é pouco desenvolvido em Threristicus caudatus (Pr9 - Fig. 15) e está presente apenas nos demais Threskiornithidae (Pr9 - Figs 11, 13 e 14).

De acordo com VeRHEYEN (1959), o osso pterigóide é longo, sendo este um padrão encontrado entre os demais Ciconiiformes, o que não corrobora com este estudo. Segundo BÜHLER (1981), o pterigóide localiza-se entre os ossos quadrado e palatino, formando assim uma "ponte" palato-quadrado. Quando sujeito a determinadas forças empregadas pelo osso quadrado, o pterigóide associado ao palatino e, em algumas espécies, ao vômer, previne a quebra/desarticulação desta "ponte" com a superfície ventral da caixa craniana.

A região rostral do pterigóide, que se articula com o osso palatino, é denominada de pé do pterigóide (pes pterygoidei) (BAumel \& Witmer 1993), sendo conspícua em P. ajaja. Observase uma fossa rasa localizada lateralmente na face rostral do osso pterigóide somente em $P$. ajaja.

Dorsalmente, o osso pterigóide possui um estreitamento 
formando uma crista 6 que separa a face lateral da face caudal; medialmente há uma crista 7 que separa a face caudal da ventral. A face caudal apresenta um sulco na porção rostromedial, compreendendo metade de sua extensão. Ainda nesta face, pode-se constatar uma fossa profunda, localizada caudalmente, com um forâmen característico.

Os Threskiornithidae apresentam as cristas 6 e 7 desenvolvidas, contudo não há muita variação entre as especiés. Em C. atratus, estas cristas mostram algumas modificações, pois a crista 6 une-se com a crista 7 na metade de extensão da face caudal do pterigóide e ambas orientam-se caudalmente. As duass cristas não foram observadas em $P$. ruber, e a crista 7 está ausente em A. cocoi.

O sulco observado na face caudal do pterigóide estendese por quase a metade da extensão da face caudal do pterigóide em P. ajaja, podendo atingir toda a sua extensão em Threristicus caudatus. Não há diferença significativa entre os Ardeidae e Ciconiidae. Não se observa este sulco em C. atratus e $P$. ruber.

Há uma fossa na face caudal do pterigóide observada apenas em $P$. ajaja e Threristicus caudatus, estando ausente em E. ruber e M. cayennensis. Não se observa esta fossa na maioria das espécies analisadas, com exceção de T. lineatum, E. thula, C. maguari e J. mycteria. Um forâmem característico nesta região está presente apenas em P. ajaja entre os Threskiornithidae; está presente também em E. thula, C. maguari e J. mycteria.

Em P. ajaja o arco jugal (J - Fig. 3) é estreito e longo, achatado dorsoventralmente na sua porção rostral e achatado lateromedialmente na sua porção mais caudal. Limita-se rostralmente com o processo jugal ( $\mathrm{PrJ}$ - Fig. 3) do osso maxilar e articula-se caudalmente com o osso quadrado ( $Q$ - Fig. 3) por meio do côndilo quadrático (condylus quadraticus), que se articula com a cótila quadradojugal (cotyla quadratojugalis, cqj Fig. 29A) do osso quadrado.

Rostralmente no arco jugal, há um conspícuo processo 10 (Pr10 - Fig. 3), localizado dorsomedialmente.

Tal processo é mais desenvolvido em Threristicus caudatus (Pr10 - Fig. 9), seguido por P. ajaja (Pr10 - Fig. 3), E. ruber (Pr10 - Fig. 7) e está ausente na maioria dos indivíduos estudados de M. cayennensis, exceto no exemplar desenhado como mostra a figura 8. Este processo não é observado nos Ardeidae e nos Ciconiidae estudados, com exceção de J. mycteria onde ele é curto.

Em P. ajaja, a porção rostral do arco jugal possui medialmente a crista 8 (Cr8 - Fig. 3) e lateralmente a crista 9 (Cr9 - Fig. 3). Entre estas cristas nota-se uma fossa (F4 - Fig. 3) que se apresenta rasa em toda sua extensão.

\section{Os quadratum}

O osso quadrado é o centro de todos os movimentos cranianos: é altamente móvel e responsável pela articulação entre a caixa craniana e a mandíbula, sendo todas as articulações do tipo diartroses (Bock 1964, BüHLER 1981).

Em P. ajaja, o osso quadrado (Q - Figs 3 e 29) tem um corpo robusto e possui três processos: a) Processo orbital (Proc. orbitalis, PrOr - Figs 3 e 29): que é achatado dorsoventralmente e projeta-se obliquamente na órbita, direcionando-se rostralmente; b) Processo ótico (Proc. oticus, PrOt - Figs 3 e 29): que se projeta dorsocaudalmente e apresenta dois capítulos: capítulo ótico (capitum oticum) achatado na sua face de articulação, que se articula com a porção caudal do meato acústico externo (meatus acusticus externus) e capítulo esquamosal (capitulum squamosum), que é arredondado na sua face de articulação; articula-se com a porção ventral do processo zigomático e dorsal do processo suprameático do osso esquamosal. Na face medial deste processo (PrOt - Fig. 29), há a crista timpânica (crista tympanica, CrTy - Fig. 29) e dorsalmente a esta crista, observase o sulco pneumático (sulcus pneumaticus, spn - Fig. 29) profundo, com dois foramens; e c) Processo mandibular (Proc. mandibularis, PrMa - Figs 3 e 29) que possui quatro côndilos: côndilo lateral (condylus lateralis, CoL - Fig. 11) e o côndilo medial (condylus medialis, CoM - Fig. 11) que apresentam uma continuidade sem fusão; côndilo pterigóideo (condylus pterygoideus, CPt - Fig. 11), que se articula com a face articular quadratica do osso pterigóide; e o côndilo caudal (condylus caudalis), inconspícuo nesta espécie.

O processo orbital do osso quadrado não apresenta diferença significativa nos Threskiornithidae (PrOr - Figs 3, 7-9 e 29-31), variando somente a porção distal deste processo, que pode ser de forma retangular como em P. ajaja (PrOr - Fig. 29) e T. caudatus (PrOr - Fig. 31) ou ainda emitindo uma projeção como em E. ruber (PrOr - Fig. 30. Não há muita variação nas demais espécies estudadas, contudo é muito bem desenvolvido nos Ardeidae.

Somente os Threskiornithidae apresentam a crista timpânica do processo ótico do quadrado; já o sulco pneumático é observado nos Threskiornithidae, Ardeidae, Ciconiidae e P. ruber. Nos Ciconiidae estudados, o sulco pneumático localiza-se distalmente na face ventral do processo ótico do quadrado.

Em relação ao processo mandibular, todos os côndilos são conspícuos nos Threskiornithinae em geral, sendo que os côndilos lateral e caudal (CoL e CoC - Figs 13 e 15) apresentam uma continuidade, assim como nos Ciconiidae, diferindo do observado em $P$. ajaja.

Na face medial do corpo do osso quadrado (corpus ossi quadrati), observa-se uma crista 10 (Cr10 - Fig. 29), que divide o corpo do quadrado; dorsalmente a esta, nota-se uma fossa 6 característica (F6 - Fig. 29) que separa o corpo do quadrado do processo orbital; tais estruturas estão ausentes nos Threskiornithinae. A crista 10 está presente em C. atratus, mas está ausente nos Ardeidae, Ciconiidae e P. ruber.

O presente estudo corrobora com as descrições de Verheyen (1959) quanto ao osso quadrado. Tal autor relata que em Threskiornithidae, Scopidae e Phoenicopteridae o osso quadrado apresenta-se mais volumoso e o processo orbital é relativamente mais longo, atingindo seu máximo de desenvolvimento nos Ardeidae; o osso quadrado é compacto nos Ciconiidae, mas principalmente nos Balaenicepidae onde o processo orbital é proporcionalmente mais curto. 

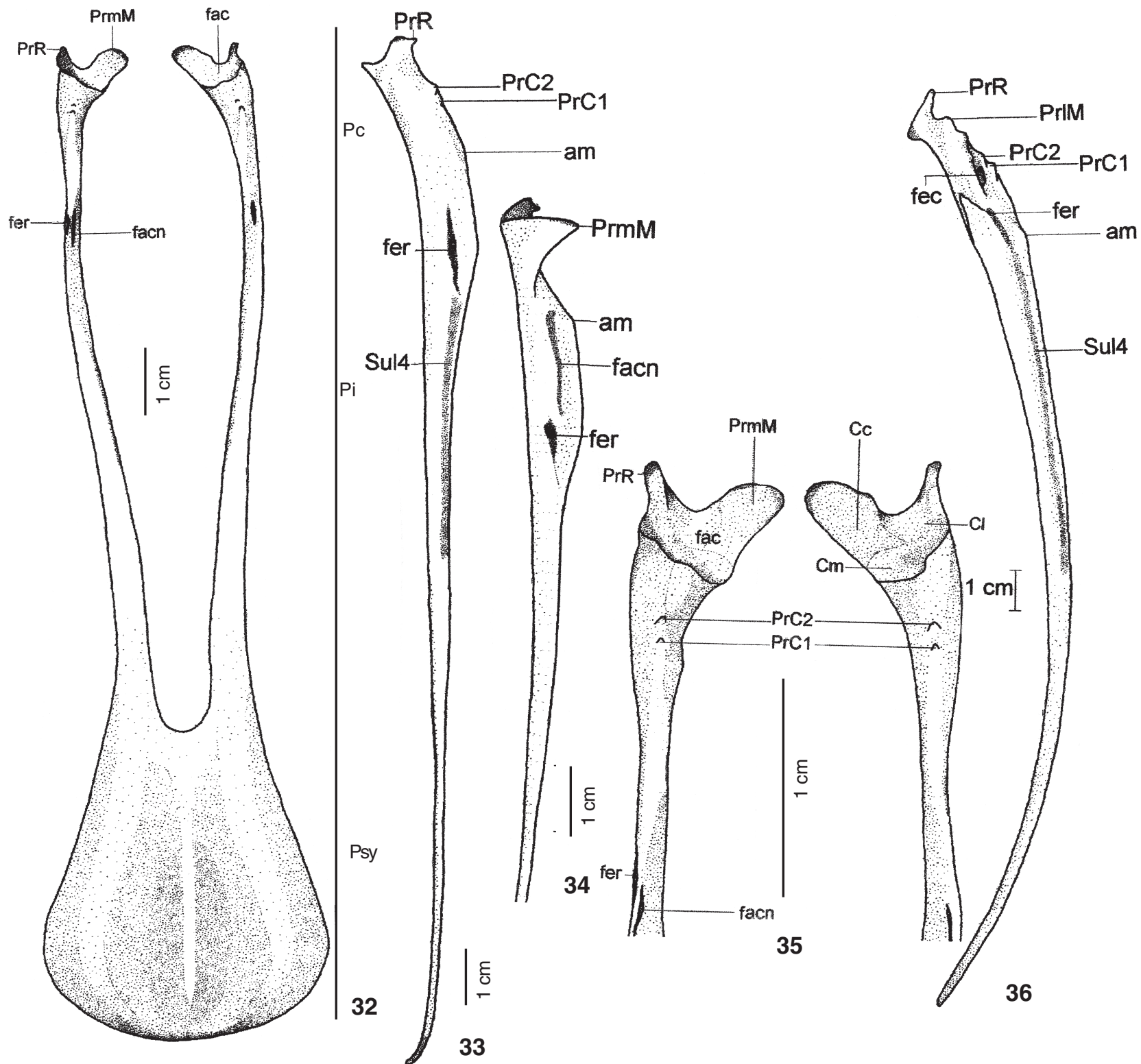

Figuras 32-36. Mandíbula: (32) Platalea ajaja, vista dorsal; (33) Platalea ajaja, vista lateral; (34) Platalea ajaja, vista medial; (35) Platalea ajaja, vista dorsocaudal; (36) Eudocimus ruber, vista lateral. (am) Ângulo da mandíbula, (Cc) cótila caudal, $(\mathrm{Cl})$ cótila lateral, $(\mathrm{Cm})$ cótila medial, (fac) fossa articular quadrática, (facn) fossa ádito canal neurovascular, (fec) fenestra caudal da mandíbula, (Pc) parte caudal, (Pi) parte intermediária, ( $\operatorname{PrC} 1$ e 2) processos coronóides, (PrIM) processo lateral da mandíbula, (PrmM) processo medial da mandíbula, (PrR) processo retroarticular, (Psy) parte sinfisiária, (Sul 4) sulco 4.

\section{Ossa Mandibulae}

A mandíbula (ossa mandibulae) de P. ajaja divide-se em três partes: a) Parte sinfisiária (pars symphisialis, Psy - Fig. 32): que reúne os dois ramos da mandíbula e mostra uma forma ampla, achatada dorsoventralmente, como uma colher, apresentando um curvamento em sua extremidade. Vários cana- lículos (foramina neurovascularia e foveae corpusculorum nervosorum) são observados nesta região nas faces dorsal e ventral e há, ainda, uma linha mediana dividindo as duas hemimandíbulas. Esta porção atinge cerca de $1 / 3$ do comprimento total da mandíbula; b) Parte intermédia (pars intermedia, Pi - Fig. 32): que corresponde à porção intermediária da mandíbula, com- 
posta principalmente pelo osso dental e atinge quase a metade do comprimento da mandíbula. É achatada dorsoventralmente na sua porção rostral (2/3) e lateromedialmente na sua porção caudal (1/3). A face lateral da sua porção caudal tem um sulco raso (Sul4 - Fig. 33) que se dirige rostralmente; c) Parte caudal (pars caudalis, Pc - Fig. 32): que é a porção caudal da mandíbula, é achatada lateromedialmente, onde se observa na sua face lateral (facies lateralis) a fenestra rostral da mandíbula (fenestra rostralis mandibulae, fer - Fig. 33), que é longa e atinge cerca da metade do comprimento desta parte da mandíbula. Observa-se ainda o ângulo da mandíbula (am - Fig. 33), que se localiza dorsalmente. Na face medial (facies medialis), pode-se observar a conspícua fossa adito canal neurovascular (fossa aditus canalis neurovascularis, facn - Fig. 13), longa e profunda; ventralmente situa-se a fenestra rostral da mandíbula (fenestra rostralis mandibulae, fer - Fig. 34). Dorsalmente apresenta os processos coronóides 1 e 2 (proc. coronoideum, PrC1 e PrC2 - Figs 33 e 35): o primeiro é mais rostral e discreto que o segundo, que é conspícuo, podendo ainda ocorrer variação individual.

As hemimandíbulas dos Threskiornithinae são achatadas lateromedialmente e afilam-se na região da sínfise mandibular, ao contrário de $P$. ajaja, que têm uma forma de colher e na parte caudal, são achatadas lateromedialmente.

A fenestra rostral da mandíbula está presente nos Threskiormithidae (fer - Figs 33, 36 e 37), Ciconiidae e demais espécies com exceção de $P$. ruber; a fenestra caudal da mandíbula está presente somente em E. ruber (fec - Fig. 36), N. nycticorax, C. maguari e $M$. americana.

Em M. cayennensis há um conspícuo tubérculo pseudotemporal (tuberculum pseudotemporale), localizado na face medial da mandíbula, podendo ser observado em alguns indivíduos de Threristicus caudatus, contudo pouco conspícuo; ele ocorre também em A. cocoi, T. lineatum, A. alba e em apenas um indivíduo de $N$. nycticorax, dentre os dois analisados. Tal tubérculo é ausente em E. ruber, P. ajaja e nas demais espécies.

Os processos coronóides 1 e 2 são conspícuos em Threristicus caudatus (PrC1 e PrC2, Figs 37 e 40), E. ruber (PrC1 e PrC2 - Figs 36 e 38) e M. cayennensis (PrC1 e PrC2 - Fig. 39); em P. ajaja (PrC1 e PrC2 - Figs 33 e 35) o primeiro é pouco evidente e o segundo é conspícuo, apresentando ainda variação individual. O processo coronóide 1 é conspícuo em $A$. cocoi e $T$. lineatum e menos conspícuo em A. alba, B. striatus, E. thula, N. nycticorax e nos Ciconiidae estudados. Nas demais espécies não se observa muita variação. O processo coronóide 2 está ausente em $C$. atratus; todavia, mostra-se conspícuo em A. cocoi e T. lineatum, seguidos por A. alba, B. striatus, E. thula, N. nycticorax, C. maguari, M. americana, J. mycteria e P. ruber.

$\mathrm{Na}$ face dorsal da parte caudal da mandíbula, há a fossa articular quadratica (fossa articularis quadratica, fac - Figs 32 e 35), que é dividida em: cótila caudal (cotyla caudalis, Cc - Fig. 35), que se articula com o côndilo caudal (condylus caudalis) do osso quadrado; cótila lateral (cotyla lateralis, Cl - Fig. 35), que se articula com o côndilo lateral (condylus lateralis) do osso quadrado; e cótila medial (cotyla medialis, Cm - Fig. 35) que se articula com o côndilo medial (condylus medialis) do quadrado.

Ainda na região caudal da mandíbula observa-se o processo medial da mandíbula (proc. medialis mandibulae, PrmM Figs 32 e 35), projetado dorsomedialmente, sendo bem desenvolvido em $P$. ajaja. Caudalmente, há o curto processo retroarticular (proc. retroarticularis, PrR - Figs 32 e 35).

O processo medial da mandíbula é bem mais desenvolvido em P. ajaja (PrmM - Fig. 35) que em M. cayennensis (PrmM Fig. 39), Threristicus caudatus (PrmM - Fig. 40) e E. ruber (PrmM - Fig. 38), onde é menos evidente. Apresenta-se bem desenvolvido em $P$. ruber, seguido pelos Ciconiidae; nos Ardeidae é pouco conspícuo.

O processo lateral da mandíbula está presente nos Threskiornithinae (PrlM, Figs 38-40), apresentando-se bem desenvolvido; não se observa tal processo em $P$. ajaja. Ele é conspícuo em A. cocoi e T. lineatum, evidente em A. alba, B. striatus, E. thula, N. nycticorax e C. maguari, seguidos por M. americana e J. mycteria; está ausente nas demais espécies.

Já o processo retroarticular é curto em P. ajaja (PrR - Fig. 35), sendo bem desenvolvido em E. ruber (PrR - Fig. 38), atingindo seu máximo comprimento em Threristicus caudatus (PrR - Fig. 40) e M. cayennensis (PrR - Fig. 39). Apresenta-se também conspícuo em $P$. ruber. Este fato não foi constatado por VERHEYEN (1959). Tal autor relata que nos Threskiornithidae, o processo retroarticular é caracteristicamente bem curto, principalmente nos Plataleinae, menos desenvolvido em Lophotibis e mesmo ausente em Hagedashia. Porém, Воск (1964) discorda, afirmando ser este processo bem desenvolvido nos Ciconiiformes, Galliformes, Psittaciformes, Anseriformes e Phoenicopteridae.

Em $P$. ajaja encontra-se a profunda fossa caudal (fossa caudalis, fc - Fig. 41) na face caudal da mandíbula, local de inserção dos componentes do músculo depressor da mandíbula ( $m$. depressor mandibulae). Esta fossa está limitada medialmente pela crista medial da mandíbula (CrmM - Fig. 41) e lateralmente pela crista lateral da mandíbula (CrlM - Fig. 41). Ventralmente estas duas cristas unem-se formando o vértice do angular (va - Fig. 41).

A fossa caudal é mais profunda em M. cayennensis (fc Fig. 43) e E. ruber (fc - Fig. 42) que em P. ajaja (fc - Fig. 41) e Threristicus caudatus (fc - Fig. 44). Os Ardeidae apresentam-na rasa, assim como em Ciconiidae, sendo quase inexistente em $J$. mycteria. Em C. atratus e P. ruber a fossa caudal é inconspícua.

As cristas lateral e medial da mandíbula são bem desenvolvidas nos Threskiornihidae (CrlM e CrmM - Figs 41-44); apresentam-se inconspícuas nos Ardeidae, exceto em N. nycticorax. Nas demais espécies, estas cristas não são conspícuas.

\section{CONCLUSÕES}

A partir do presente estudo, pode-se concluir que, além de P. ajaja (Plataleinae) apresentar um característico formato do bico (em forma de colher), características esta que a separa dos Threskiornithinae, outras características importantes foram 

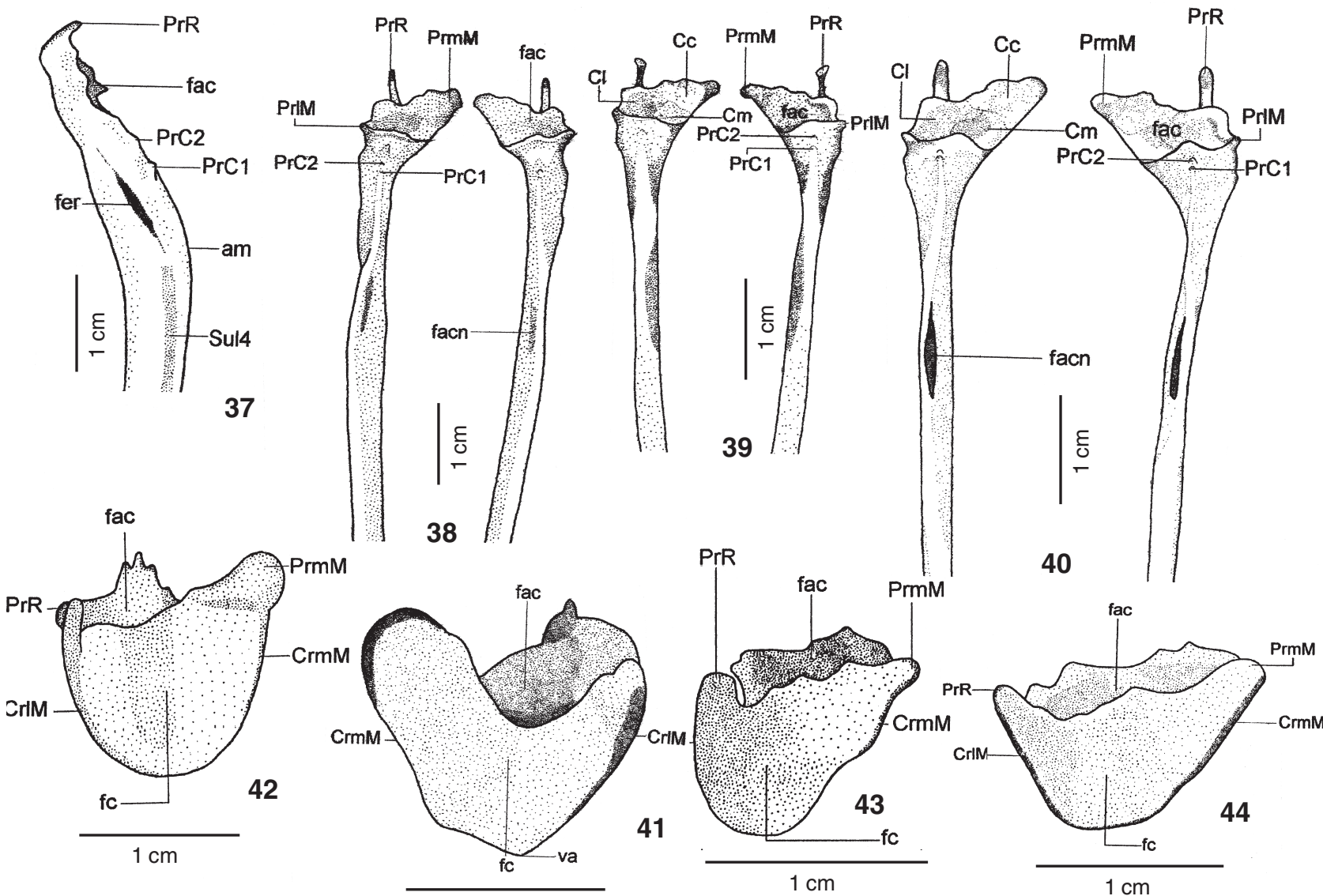

$1 \mathrm{~cm}$

Figuras 37-44. Mandíbula: (37) Theristicus caudatus, vista lateral; (38) Eudocimus ruber, vista dorsocauual; ( गy) Iviesembrinibis cayennensis, vista dorsocaudal; (40) Theristicus caudatus, vista dorsocaudal; (41) Platalea ajaja, vista caudal; (42) Eudocimus ruber, vista; (43) Mesembrinibis cayennensis, vista caudal; (44) Theristicus caudatus, vista caudal. (am) Ângulo da mandíbula, (Cc) côndilo caudal, (Cl) côndilo lateral, $(\mathrm{Cl})$ cótila lateral, $(\mathrm{Cm})$ côndilo medial, $(\mathrm{Cm})$ cótila medial, $(\mathrm{CrIM})$ crista lateral da mandíbula, (CrmM) crista medial da mandíbula, (fac) fossa articular quadrática, (facn) fossa ádito canal neurovascular, (fc) fossa caudal, (fer) fenestra rostral da mandíbula, (PrC 1 e 2) processos coronóides, (PrIM) processo lateral da mandíbula, (PrmM) processo medial da mandíbula, (PrR) processo retroarticular, (Sul 4) sulco 4, (va) vértice do angular.

observadas somente nesta espécie. São elas: sutura frontonasal conspícua; processo supra-orbital do osso lacrimal curto; processo lateral do paraesfenóide conspícuo e afilado na extremidade; narina curta e de forma oval; processo retroarticular curto; projeção do paraesfenóide curta; mandíbula achatada dorsoventralmente e alargada na sua extremidade.

Todavia, constatou-se que $P$. ajaja compartilha com os demais Threskiornithidae estudados as seguintes características: a não-fusão dos ossos frontal e lacrimal; lacrimal bem desenvolvido; processo zigomático bem desenvolvido; presença dos fontículos occipitais; processo paraoccipital bem desenvolvido; narina localizada em um sulco do osso premaxilar; região do maxilar reduzido; e vômer conspícuo e achatado laterolateralmente.
Ainda no presente estudo, observou-se também algumas características presentes somente nos Threskiornithinae estudados, como a concavidade localizada rostromedialmente nas regiões do frontal e nasal; um longo processo supra-orbital do lacrimal; projeção do paraesfenóide muito bem desenvolvida; uma desenvolvida projeção 1 do ectetmóide; presença de um processo 6 no lateroesfenóide; maxila superior estreita, curvada e achatada laterolateralmente; narina comprida e estreita; processo retroarticular bem desenvolvido; e presença do processo lateral da mandíbula.

Entretanto, são necessários estudos osteológicos que conteplem as outras espécies da família, bem como um estudo filogenético do grupo para maior compreensão das relações existentes entre os Threskiornithidae. 


\section{AGRADECIMENTOS}

À Dra. Maria Luiza Videira Marceliano, curadora da coleção de aves do Museu Paraense Emílio Goeldi, pelo empréstimo do material e pelas valiosas sugestões, à CAPES pelo financiamento da pesquisa e aos Profs. Drs. Elizabeth Höfling e Carlos Alberto Vicentini pelas primorosas sugestões e correções.

\section{REFERÊNCIAS BIBLIOGRÁFICAS}

American Ornithologists' Union 1998. Check-list of North American Birds. Washington, American Ornithologists' Union, $7^{\text {th }}$ ed., $829 \mathrm{p}$.

Austin JR., O.L. \& A. Singer. 1983. Birds of the world. Verona, Ed. Optimum Books, 319p.

Baumel, J.J.; L.M. Witmer. 1993. Osteologia, p.45-132. In: J.J. Baumel; A.S. King; J.E. Breazile; H.E. Evans; J.C. Berge (Eds). Handbook of avian anatomy: Nomina Anatomica Avium. Cambridge, Nutall Ornitological Club, $2^{\text {nd }}$ ed., XXIV+779p.

Bоск, W.J. 1964. Kinects of the avian skull. Journal of Morphology, Philadelphia, 114: 1-42.

BüHLER, P. 1981. Functional anatomy of the avian jaw apparatus, p. 439-468. In: A.S. KING \& J. McLleland (Eds). Form and Function in birds. London, Academic Press, vol. 2, 496p.

Cracraft, J. 1968. The lacrimal-ectethmoid bone complex in birds: a single acharacter analysis. American Midland Natural, New York, 80 (2): 316-359.

Cracraft, J. 1981. Toward a phylogenetic classification of the recent birds of the world (Class Aves). The Auk, Washington, 98: 681-714.

Donatelli, R.J. 1997. Osteologia e miologia craniana de Dendrocolaptidae (Passeriformes, Tyranni). 1. Gêneros Glyphorhynchus, Capylorhamphus, Dendrocincla, Xiphorhynchus e Dendrocolaptes. Ararajuba, Londrina, 5 (1): 19-37.

GADOw, H. 1893. Vögel II. Systematischer Theil. In: C.F. Winter (Ed.). Bronn's Klassen und Ordnungen des Thier-Reichs. Leipzig, vol. 6, 303p.

Höfling, E.; J.P. GaSc. 1984. Biomécanique du crâne et du bec chez Ramphastos (Aves: Ramphastidae). Gegenbaurs Morphology Jahrb., Leipizig, 130: 125-147.

Howard, R. \& A. MOORE. 1998. A complete checklist of the birds on the world. London, Academic Press, $2^{\text {nd }}$ ed., 630p.

Huxley, T.H. 1867. On the classification of birds and on the taxonomic value of the cranial bones observable in that class.
Proceedings of Zoology Society, London, 1867: 415-472.

Jollie, M. 1957. The head skeleton of the chicken and remarks on the anatomy of this region in other birds. Journal of Morphology, Philadelphia, 100 (4): 389-436.

KAHL, M.P.E.G. 1979. Family Coconiidae, p. 245-252. In: E. MAYR $\&$ G.W. Cottrell (Eds). Check list of birds of the world. Cambridge, Museu of Comparative Zoology, vol. 1., $2^{\text {nd }}$ edition, 374p.

Matheu, E. \& J. Del Hoyo. 1992. Family Threskiornithidae (Ibises and Spoonbills), p. 472-506. In: J. Del Hoyo; A. Elloto (Eds). Handbook of the Birds of the World. Barcelona, Lynx Edicions, vol.1, 696p.

Mitchell, P.C. 1913. Observations on the anatomy of the shoebill (Balaeniceps rex) and allied birds. Proceedings of Zoology of Society London, London, 1913: 644-703.

Monroe, B.L. \& C.G. Sibley. 1993. A world checklist of birds. London, Yale University Press, 393p.

PAYNE, R.B.; C.J. Risley. 1976. Systematics and evolutionary relationships among the Herons (Ardeidae). Miscellany Publications of The Museum of Zoology, Michigan, 50: 1-115.

Pycraft, W.P. 1903. Contribuitions to the osteology of birds. Proceedings of Zoology of Society London, London, 1: 258-291.

Reichenow, A. 1882. Die Vögel der Zoologischen Gärten. Leipzig, L.A. Kittler, 278p.

SеEвонм, H. 1895. Classification of birds: an attempt to diagnose the subclasses, orders, suborders and families of existing birds. London, R.H. Porter, Supplement, 76p.

Sharpe, R.B. 1899. A hand-list of the genera and species of birds. London, British Museum, vol. 1, 83p.

Sibley, C.G. \& J.E. Ahlquist. 1972. A comperative study of the egg-white proteins of non-passarine birds. Bulletin Peabody Museum of Natural History, New Haven, 39: 1-279.

SICK, H. 1997. Ornitologia Brasileira. Rio de Janeiro, Ed. Nova Fronteira, 862p.

SunDEVALL, C.J. 1872. Methodi naturalis avium disponendarum tentamem. Stockholm, Samson and Wallin, 98p.

Verheyen, R. 1959. Note sur la systématique de bases des Lariformes. Bulletin Institute Royal Science Natural Belgium, Bruxelles, 35 (9): 1-16.

Verheyen, R. 1961. A new classification for the non-passerine birds of the world. Bulletin Institute Royal Science Natural Belgium, Bruxelles, 37 (27): 1-36.

Recebido em 09.XI.2004; aceito em 30.VIII.2005. 